\title{
THE T-GRAPH OF A MULTIGRADED HILBERT SCHEME
}

\author{
MILENA HERING AND DIANE MACLAGAN \\ Dedicated to the memory of Mikael Passare
}

\begin{abstract}
The $T$-graph of a multigraded Hilbert scheme records the zero and one-dimensional orbits of the $T=\left(K^{*}\right)^{n}$ action on the Hilbert scheme induced from the $T$-action on $\mathbb{A}^{n}$. It has vertices the $T$-fixed points, and edges the onedimensional $T$-orbits. We give a combinatorial necessary condition for the existence of an edge between two vertices in this graph. For the Hilbert scheme of points in the plane, we give an explicit combinatorial description of the equations defining the scheme parameterizing all one-dimensional torus orbits whose closures contain two given monomial ideals. For this Hilbert scheme we show that the $T$-graph depends on the ground field, resolving a question of Altmann and Sturmfels.
\end{abstract}

\section{INTRODUCTION}

The Hilbert scheme of points in the plane is a classical and well-studied space, and an important technique in its study is to consider the fixed points of the action of the torus $\left(K^{*}\right)^{2}$ on the Hilbert scheme. However there is no known combinatorial condition deciding whether two fixed points, which correspond to monomial ideals in $K[x, y]$, lie in the closure of a one-dimensional torus orbit. In this paper we give a necessary combinatorial condition in the more general context of multigraded Hilbert schemes.

The multigraded Hilbert scheme, introduced by Haiman and Sturmfels in [HS04], parameterizes subschemes $Z$ of $\mathbb{A}^{n}$ invariant under the action of an abelian group for which $H^{0}\left(\mathcal{O}_{Z}\right)$ has a prescribed decomposition into irreducible representations. Equivalently, $\mathrm{Hilb}_{S}^{h}$ parameterizes all ideals $I$ in the polynomial ring $S=K\left[x_{1}, \ldots, x_{n}\right]$ that are homogeneous with respect to a grading by an abelian group $A$, and have a fixed multigraded Hilbert function $h: A \rightarrow \mathbb{N}$ given by $h(a)=\operatorname{dim}_{K}(S / I)_{a}$. Specific examples of multigraded Hilbert schemes include the Grothendieck Hilbert scheme of subschemes of projective space, Hilbert schemes of points in affine space, and $G$-Hilbert schemes for abelian groups $G$.

The action of $T=\left(K^{*}\right)^{n}$ on $\mathbb{A}^{n}$ induces an action of $T$ on $\operatorname{Hilb}_{S}^{h}$ whose fixed points are the monomial ideals in $\operatorname{Hilb}_{S}^{h}$. The $T$-graph of the multigraded Hilbert scheme Hilb $_{S}^{h}$ has vertices these fixed points, and an edge between two vertices $M$ and $N$ if there is a one-dimensional torus orbit whose closure contains $M$ and $N$. This is closely related to the graph of monomial ideals of Altmann and Sturmfels [AS05.

The main result of this paper is a necessary condition (Theorem 1.3) for two vertices $M$ and $N$ to be connected by an edge of the $T$-graph. In the case of $\operatorname{Hilb}^{d}\left(\mathbb{A}^{2}\right)$ we give a combinatorial description (Theorem 1.4 ) over $\mathbb{Z}$ for the equations of the edgeschemes describing all $T$-orbits joining a pair of fixed points. 
One motivation to study the $T$-graph is to understand the connectedness of multigraded Hilbert schemes. In contrast to the classical Hilbert scheme of subschemes of projective space, which is always connected [Har66], multigraded Hilbert schemes can be disconnected SSan05]. However, a necessary and sufficient condition for a multigraded Hilbert scheme to be connected (when the grading is positive and $K=\mathbb{C}$ ) is for the T-graph of Hilb $\mathrm{H}_{S}^{h}$ to be connected; see [AS05, Corollary 16]. The sufficiency has been well-exploited in the literature (see [PS05], MS10]), and we hope that through a better understanding of the $T$-graph the necessity can be used to exhibit more tractable examples of disconnected multigraded Hilbert schemes. Another motivation comes from the use of $T$-graphs of varieties to understand cohomology. The standard set-up of [GKM98] to compute cohomology from the T-graph of a variety requires that the one-dimensional orbits be isolated, which need not be the case for multigraded Hilbert schemes. However one could still hope to deduce information about the cohomology in these cases; see for example [BCS08, Eva07].

Monomial ideals are fundamentally combinatorial objects, and a natural question is whether the $T$-graph has a purely combinatorial description. The main results of this paper illustrate the complexity of this question. Since the one-dimensional orbits are not isolated, we consider the edge-scheme $E(M, N)$ parameterizing all ideals $I \in \mathrm{Hilb}_{S}^{h}$ lying in a one-dimension $T$-orbit whose closure contains $M$ and $N$. In Example 2.11 we construct an example of an edge-scheme in $\operatorname{Hilb}^{10}\left(\mathbb{A}^{2}\right)$ that has $\mathbb{R}$-valued points, but no $\mathbb{Q}$-valued points. This shows that the $T$-graph depends on the field $K$, solving a problem posed by Altmann and Sturmfels in [AS05, Section 5]. It also shows that there cannot be a purely combinatorial description of the generators of an ideal $I$ contained in a one-dimensional $T$-orbit.

Our first step towards a combinatorial necessary condition for the existence of an edge in the $T$-graph is to show that we can reduce to a simpler multigraded Hilbert scheme whose Hilbert function has finite support, so $\sum_{a \in A} h(a)<\infty$. More precisely, we show that if there is an edge in the $T$-graph of $\mathrm{Hilb}_{S}^{h}$ between two monomial ideals $M$ and $N$ then there exists a positive grading of the polynomial ring $S$ by $\mathbb{Z}^{n} / \mathbb{Z} \mathbf{c}$ for some $\mathbf{c} \in \mathbb{Z}^{n}$, and a Hilbert function $H: \mathbb{Z}^{n} / \mathbb{Z} \mathbf{c} \rightarrow \mathbb{N}$ such that $M$ and $N$ have Hilbert function $H$, and there is an edge in the $T$-graph between $M$ and $N$ in this refined multigraded Hilbert scheme, which we denote by $\mathbb{H}_{\mathbf{c}}(H)$. See Corollary 2.6.

The following theorem, which holds over an arbitrary base, gives the reduction to finite support Hilbert functions.

Theorem 1.1. Let $h: A \rightarrow \mathbb{N}$ be a Hilbert function. If the A-grading of $S$ is positive, then there exists $\bar{h}: A \rightarrow \mathbb{N}$ with $\sum_{a \in A} \bar{h}(a)<\infty$ and an isomorphism

$$
\operatorname{Hilb}_{S}^{h} \cong \operatorname{Hilb}_{S}^{\bar{h}} .
$$

This isomorphism respects the T-action on the two Hilbert schemes.

Our combinatorial necessary condition uses the following definition of an arrow map. It is a modification of the definition of a "system of arrows" introduced by Evain in [Eva04 to study incidence conditions for Białynicki-Birula cells in multigraded Hilbert schemes of points in the plane; see Remark 4.9. 
Definition 1.2. Let $S$ be graded by $\mathbb{Z}^{n} / \mathbb{Z} \mathbf{c}$ for some $\mathbf{c} \in \mathbb{Z}$, and let $\prec$ be a monomial term order on $S$. For a monomial ideal $M$, let $\operatorname{Mon}(M)$ denote the set of monomials in $M$. If two monomials $m=x^{u}$ and $m^{\prime}=x^{v}$ have the same degree, then $u-v=\ell \mathbf{c}$, and we define the distance between $m$ and $m^{\prime}$ to be $d\left(m, m^{\prime}\right)=|\ell|$. For two monomial ideals $M$ and $N$, we say that $f: \operatorname{Mon}(M) \rightarrow \operatorname{Mon}(N)$ is an arrow map if

(1) $f$ is a degree-preserving bijection such that $m \succeq f(m)$ for all $m \in \operatorname{Mon}(M)$;

(2) for all $m \in \operatorname{Mon}(M)$ and all multiples $m^{\prime}$ of $m$, we have

$$
d\left(m^{\prime}, f\left(m^{\prime}\right)\right) \leqslant d(m, f(m)) ;
$$

(3) for all $m \in \operatorname{Mon}(N)$ and all multiples $m^{\prime}$ of $m$, we have

$$
d\left(f^{-1}\left(m^{\prime}\right), m^{\prime}\right) \leqslant d\left(f^{-1}(m), m\right) .
$$

See Example 3.1 and Figure 1 for an illustration of this concept.

Theorem 1.3. Assume that $H: \mathbb{Z}^{n} / \mathbb{Z} \mathbf{c} \rightarrow \mathbb{N}$ has finite support, and let $M, N$ be monomial ideals in $\mathbb{H}_{\mathbf{c}}(H)$ that are connected by an edge in the $T$-graph of $\mathbb{H}_{\mathbf{c}}(H)$.

(1) There exists an arrow map $f: M \rightarrow N$ with respect to some term order $\prec$.

(2) Fix $r_{1}, \ldots, r_{n}$ such that $x_{i}^{r_{i}} \in M \cap N$ for all $1 \leqslant i \leqslant n$, and let $Q=$ $\left\langle x_{1}^{r_{1}}, \ldots, x_{n}^{r_{n}}\right\rangle$. Then there also exists an arrow map $\widehat{f}: \operatorname{Mon}((Q: M)) \rightarrow$ $\operatorname{Mon}((Q: N))$ with respect to the same term order as in (1).

Condition (1) holds without the condition that the Hilbert function has finite support; see Corollary 3.3. It is not sufficient for the existence of an edge in the $T$-graph; see Example 3.7. We do not know, however, of an example showing that both (1) and (2) together do not suffice to guarantee the existence of an edge. For the Hilbert scheme of $d$ points in the plane, these conditions are sufficient for $d \leqslant 16$; see Table 5.4. On the other hand, the proof of Theorem 1.3 is based on associating an arrow map to an ideal $I$ in the $T$-orbit (Proposition 3.2 ), and we have examples of arrow maps that are not associated to ideals (Example 3.6).

In the case of the Hilbert scheme of points in the plane, there exists an explicit combinatorial description of the equations for the edge-scheme describing all onedimensional $T$-orbits joining a fixed pair of monomial ideals. In particular, this scheme is defined over $\mathbb{Z}$.

Theorem 1.4. Let $M, N$ be monomial ideals in $K[x, y]$ with the same Hilbert function with respect to a positive $\mathbb{Z}^{2} / \mathbb{Z} \mathbf{c}$-grading. The ideal of the edge-scheme $E(M, N)$ is generated by polynomials $F_{n, s}$ with integer coefficients, where $n$ is a minimal generator of $N$, and $s$ is a standard monomial of $M$ with $\operatorname{deg}(s)=\operatorname{deg}(n)$.

The terms of the polynomials $F_{n, s}$ have an explicit combinatorial form in terms of the torus weights of the action of the torus on the tangent spaces to $M$ and $N$ in $\mathbb{H}_{\mathbf{c}}(H)$ that we describe in detail in Section 4.2. The equations are obtained by combining an algorithm of Altmann and Sturmfels [AS05, Algorithm 5] with a description, due to Evain [Eva04, of the Białynicki-Birula cells in this Hilbert scheme.

This paper is partially experimental in nature, and we relied heavily on computations using the computer algebra system Macaulay 2 [GS]. The resulting code is available from the second author's webpage as the Macaulay 2 package TEdges 
[Mac11]. Some details of these computations are given in the last section of the paper.

Acknowledgments We thank Bernd Sturmfels for stimulating our interest in the $T$-graph. This paper was written at several mathematical institutes and we are grateful to the Institute of Mathematics and its Applications, the Mathematical Sciences Research Institute, the Mathematisches Forschungsinstitut Oberwolfach, and the Mittag Leffler institute for their hospitality. The first author was partially supported by an Oberwolfach Leibniz Fellowship and NSF grant DMS 1001859. The second author was partially supported by EPSRC grant EP/I008071/1.

\section{Reduction to THE POSITIVELy GRADED ARTINiAn CASE}

In this section we show that the study of the $T$-graph of arbitrary multigraded Hilbert schemes can be reduced to the study of multigraded Hilbert schemes parameterizing finite-length ideals that are homogeneous with respect to a positive grading by $\mathbb{Z}^{n} / \mathbb{Z} \mathbf{c}$, where $\mathbf{c} \in \mathbb{Z}^{n}$. Moreover, we show that every positively-graded multigraded Hilbert scheme is isomorphic to some multigraded Hilbert scheme parameterizing finite-length ideals. Throughout this section $S$ denotes the polynomial ring $S=K\left[x_{1}, \ldots, x_{n}\right]$, where unless otherwise noted $K$ is a field.

\subsection{Multigraded Hilbert schemes.}

Definition 2.1 ([HS04] $)$. Fix a grading by an abelian group $A$ on $S$, and a function $h: A \rightarrow \mathbb{N}$. The multigraded Hilbert scheme $\mathrm{Hilb}_{S}^{h}$ parameterizes all homogeneous ideals $I$ in $S$ with Hilbert function $\operatorname{dim}_{K}(S / I)_{a}=h(a)$ for all $a \in A$.

The multigraded Hilbert scheme $\operatorname{Hilb}_{S}^{h}$ is a quasiprojective scheme over $\operatorname{Spec}(K)$; see [HS04, Theorem 1.1]. Following [HS04, we say that the grading is positive if $\operatorname{dim}_{K} S_{a}<\infty$ for all $a \in A$. In this case Hilb His $_{S}^{h}$ is projective; see [HS04, Corollary $1.2]$.

Example 2.2. (1) Fix an integer-valued polynomial $P$. There exists $D \gg 0$ such that when $A=\mathbb{Z}, h(a)=0$ for $a<0, h(a)=\operatorname{dim}_{K} S_{a}$ for $0 \leqslant a<D$, and $h(a)=P(a)$ for $a \geqslant D$, then Hilb $_{S}^{h}$ is Grothendieck's Hilbert scheme $\operatorname{Hilb}^{P}\left(\mathbb{P}^{n-1}\right)$ parameterizing all subschemes of $\mathbb{P}^{n-1}$ with Hilbert polynomial $P[\underline{\mathrm{HS} 04}, \S 4]$.

(2) When $A=0$, and $h(0)=d$, then $\operatorname{Hilb}_{S}^{h}$ is the Hilbert scheme $\operatorname{Hilb}^{d}\left(\mathbb{A}^{n}\right)$ of $d$ points in $\mathbb{A}^{n}$.

(3) For arbitrary $A$, if $h(a)=1$ whenever $\operatorname{dim}_{K} S_{a}>0$ and $h(a)=0$ otherwise, then $\mathrm{Hilb}_{S}^{h}$ is the toric Hilbert scheme [PS02], [HS04, §5]. When $A$ is finite this is Nakamura's G-Hilbert scheme [Nak01].

2.2. Background on the $T$-graph of a multigraded Hilbert scheme. The action of the torus $T=\left(K^{*}\right)^{n}$ on $\mathbb{A}^{n}$ induces an action on Hilb $b_{S}^{h}$ whose fixed points are the monomial ideals contained in $\operatorname{Hilb}_{S}^{h}$.

Definition 2.3. The T-graph of $\mathrm{Hilb}_{S}^{h}$ has vertices the monomial ideals in $\mathrm{Hilb}_{S}^{h}$. There is an edge joining two monomial ideals $M, N \in \operatorname{Hilb}_{S}^{h}$ if there is $I \in \operatorname{Hilb}_{S}^{h}$ such that the $T$-orbit of $I$ contains $M$ and $N$ in its closure and is one-dimensional. 
The $T$-graph has an interpretation in terms of Gröbner theory, which we now explain. For basic facts about Gröbner bases and initial ideals, see [CLO07]. For the geometric interpretation of initial ideals as limits of one-parameter torus orbits see [Eis95, Chapter 15.8].

For $\mathbf{c}=\left(c_{1}, \ldots, c_{n}\right) \in \mathbb{Z}^{n}$, we define a $\left(\mathbb{Z}^{n} / \mathbb{Z} \mathbf{c}\right)$-grading on $S=K\left[x_{1}, \ldots, x_{n}\right]$ by letting $\operatorname{deg}\left(x_{i}\right)=\mathbf{e}_{\mathbf{i}}+\mathbb{Z} \mathbf{c}$, where $\left\{\mathbf{e}_{\mathbf{1}}, \ldots, \mathbf{e}_{\mathbf{n}}\right\}$ is the standard basis of $\mathbb{Z}^{n}$. Let $\mathbf{c}^{+}=\sum_{c_{i}>0} c_{i} \mathbf{e}_{i}$ and $\mathbf{c}^{-}=\sum_{c_{i}<0}-c_{i} \mathbf{e}_{i}$, so $\mathbf{c}=\mathbf{c}^{+}-\mathbf{c}^{-}$. The grading induced by $\mathbf{c}$ is positive if and only if $\mathbf{c}^{+} \neq 0$ and $\mathbf{c}^{-} \neq 0$.

We next note that any non-monomial ideal $I$ that is homogeneous with respect to this $\mathbb{Z}^{n} / \mathbb{Z} \mathbf{c}$-grading has either exactly two initial ideals, if the grading is positive, or exactly one initial ideal otherwise. Indeed, a homogeneous polynomial has the form $f=\sum_{i=0}^{s} a_{i} x^{u+i c}$, where we assume $a_{0}, a_{s} \neq 0$. The initial term is $\operatorname{in}_{\prec}(f)=a_{0} x^{u}$ if $x^{\mathbf{c}^{+}} \prec x^{\mathbf{c}^{-}}$and $\operatorname{in}_{\prec}(f)=a_{s} x^{\mathbf{u}+s \mathbf{c}}$ if $x^{\mathbf{c}^{+}} \succ x^{\mathbf{c}^{-}}$. Thus the initial ideal of $I$ with respect to a term order $\prec$ only depends on whether $x^{\mathbf{c}^{+}} \prec x^{\mathbf{c}^{-}}$or $x^{\mathbf{c}^{+}} \succ x^{\mathbf{c}^{-}}$. If the $\mathbb{Z}^{n} / \mathbf{c} \mathbb{Z}$-grading is positive, then both $\mathbf{c}^{+}$and $\mathbf{c}^{-}$are nonzero, so a non-monomial ideal has exactly two monomial initial ideals. However, if the grading is not positive, so without loss of generality $\mathbf{c} \geqslant 0$, the monomials of degree $a$ have the form $x^{u+i \mathbf{c}}$ for some $u \in \mathbb{N}^{n}$ and $i \geqslant 0$, and the standard monomials of any monomial initial ideal in degree $a$ are $x^{u+i c}$ for $0 \leqslant i<\operatorname{dim}_{K}(S / I)_{a}$. Thus for every Hilbert function $H$ there exists exactly one monomial ideal with this Hilbert function. In particular, a homogeneous polynomial has exactly one initial ideal in this case.

Definition 2.4. Assume that the $\mathbb{Z}^{n} / \mathbb{Z} \mathbf{c}$-grading on $S$ is positive. For a term order $\prec$ with $x^{\mathbf{c}^{+}} \prec x^{\mathbf{c}^{-}}$(resp. $x^{\mathbf{c}^{+}} \succ x^{\mathbf{c}^{-}}$) we let $\prec$ opp be any term order with $x^{\mathbf{c}^{+}} \succ x^{\mathbf{c}^{-}}$ (resp. $x^{\mathbf{c}^{+}} \prec x^{\mathbf{c}^{-}}$).

Proposition 2.5. Let $M, N$ be monomial ideals in $S$. There exists a one-dimensional torus orbit $\mathcal{O} \subset \operatorname{Hilb}_{S}^{h}$ such that $\overline{\mathcal{O}}=\mathcal{O} \cup\{M, N\}$ if and only if there exists $\mathbf{c}=$ $\left(c_{1}, \ldots, c_{n}\right) \in \mathbb{Z}^{n}$ with $\mathbf{c}^{+} \neq 0, \mathbf{c}^{-} \neq 0$, a term order $\prec$, and an ideal $I$ homogeneous with respect to the $\mathbb{Z}^{n} / \mathbb{Z} \mathbf{c}$-grading such that in ${ }_{\prec} I=M$ and in ${ }_{\prec \text { opp }} I=N$. The vector c can be chosen so that the $\mathbb{Z}^{n} / \mathbb{Z} \mathbf{c}$-grading of $S$ refines the grading on $S$.

Proof. First note that an ideal $I$ is contained in a one-dimensional torus orbit if and only if $I$ is fixed by a codimension-one subtorus $T^{\prime}$. If $T^{\prime}$ is a codimension-one subtorus of $T$ and $\mathbf{c}$ is the generator of the subgroup of $M:=\operatorname{Hom}\left(T, K^{*}\right) \cong \mathbb{Z}^{n}$ that is the image of the inclusion $\operatorname{Hom}\left(T / T^{\prime}, K^{*}\right) \cong \mathbb{Z} \hookrightarrow \operatorname{Hom}\left(T, K^{*}\right)$, then $I$ is fixed by $T^{\prime}$ if and only if $I$ is homogeneous with respect to the induced $\mathbb{Z}^{n} / \mathbf{c} \mathbb{Z}$-grading; see [MS05, Lemma 10.3].

For $I \in \operatorname{Hilb}_{S}^{h}$ lying in a one-dimensional torus orbit, $I$ is also homogeneous with respect to the $A$-grading on $S$. Write $A=\mathbb{Z}^{n} / L$ for some lattice $L$; two monomials $x^{u}$ and $x^{v}$ have the same degree with respect to the $A$-grading if and only if $u-v \in L$. Choose a generating set for $I$ that is homogeneous with respect to both the $\mathbb{Z}^{n} / \mathbb{Z} \mathbf{c}$ and $A$ gradings, with the property that no summand of any generator lives in $I$. Such generators have the form $\sum a_{i} x^{u+i \mathbf{c}}$, where $i \mathbf{c} \in L$. If $j$ the greatest common divisor of all differences $i-i^{\prime}$ with $a_{i}, a_{i^{\prime}} \neq 0$, then $I$ is homogeneous with respect to the $\mathbb{Z}^{n} / \mathbb{Z} j \mathbf{c}$-grading, and $j \mathbf{c} \in L$. Thus after replacing $\mathbf{c}$ by $j \mathbf{c}$ the grading of $S$ by 
$\mathbb{Z}^{n} / \mathbb{Z} \mathbf{c}$ refines the existing grading in the sense of [HS04, p729]. It thus remains to check that $M$ and $N$ are the two initial ideals of $I$.

Let $H$ be the Hilbert function of $I$ with respect to the $\mathbb{Z}^{n} / \mathbf{c} \mathbb{Z}$-grading. The inclusion of Hilb $H_{S}^{H}$ into $\mathrm{Hilb}_{S}^{h}$ as a closed subscheme ([HS04, Proposition 1.5]) means that $M$ and $N$ also have Hilbert function $H$ with respect to the $\mathbb{Z}^{n} / \mathbf{c} \mathbb{Z}$-grading. This means that this grading is positive, as otherwise there would be only one monomial ideal with Hilbert function $H$. Thus $I$ has two initial ideals, so it remains to observe that all initial ideals of $I$ are contained in the closure of the $T$-orbit of $I$.

Let $\mathbf{w} \in \operatorname{Hom}(M, \mathbb{Z})$ and let $\lambda_{\mathbf{w}} \hookrightarrow T$ be the one-parameter subgroup associated to $\mathbf{w}$. The composition of the inclusion of the one-parameter subgroup $\lambda_{\mathbf{w}}$ into $T$ and the projection $T \rightarrow T / T^{\prime}$ is an isomorphism if and only if $\langle\mathbf{w}, \mathbf{c}\rangle \neq 0$. So if $I$ is an ideal that is fixed by $T^{\prime}$ and $\mathbf{w}$ satisfies $\langle\mathbf{w}, \mathbf{c}\rangle \neq 0$, then the orbits $T \cdot I$ and $\lambda_{\mathbf{w}} \cdot I$ are equal. In particular, their closures in $\mathrm{Hilb}_{S}^{h}$ agree. The claim now follows from the interpretation of initial ideals as flat limits of one-parameter torus orbits, as the two points $M$ and $N$ in the closure of the $T$-orbit of $I$ must be $\operatorname{in}_{\mathbf{w}}(I)$ and $\operatorname{in}_{-\mathbf{w}}(I)$ in the notation of [Eis95, Chapter 15.8]. Since $\operatorname{in}_{\mathbf{w}}(I)$ and $\operatorname{in}_{-\mathbf{w}}(I)$ are distinct monomial ideals, they equal $\operatorname{in}_{\prec}(I)$ and $\operatorname{in}_{\prec \text { opp }}(I)$ for some term order $\prec$.

It follows that in order to study one-dimensional torus orbits in any multigraded Hilbert scheme Hilb $_{S}^{h}$, it suffices to study multigraded Hilbert schemes with grading group $\mathbb{Z}^{n} / \mathbb{Z} \mathbf{c}$ and Hilbert function $H: \mathbb{Z}^{n} / \mathbb{Z} \mathbf{c} \rightarrow \mathbb{N}$. We denote the corresponding multigraded Hilbert scheme by $\mathbb{H}_{\mathbf{c}}(H)$.

Corollary 2.6. Let $M, N$ be monomial ideals in $\mathrm{Hilb}_{S}^{h}$. Then $M$ and $N$ are connected by an edge in the T-graph if and only if there exists $\mathbf{c} \in \mathbb{Z}^{n}$ and $H: \mathbb{Z}^{n} / \mathbb{Z} \mathbf{c} \rightarrow \mathbb{N}$ such that $M, N \in \mathbb{H}_{\mathbf{c}}(H)$ and there is an edge between $M$ and $N$ in the $T$-graph of $\mathbb{H}_{\mathbf{c}}(H)$.

Note that every $I \in \mathbb{H}_{\mathbf{c}}(H)$ is either a monomial ideal, or lies in a one-dimensional torus orbit.

Remark 2.7. For a given monomial ideal $M \in \operatorname{Hilb}_{S}^{h}$, there are only finitely many c such that $M$ is contained in a positive-dimensional $\mathbb{H}_{\mathbf{c}}(H)$ for some Hilbert function $H$. These vectors $\mathbf{c}$ are the weights of the torus action on the tangent space to Hilb $_{S}^{h}$ at $M$.

Recall the definition of an arrow map (Definition 1.2).

Definition 2.8. We define a partial order on the monomial ideals in $\mathbb{H}_{\mathbf{c}}(H)$ by letting $M>N$ if there exists a map $f: \operatorname{Mon}(M) \rightarrow \operatorname{Mon}(N)$ satisfying condition (1) of Definition 1.2.

This partial order was used in Yaméogo Yam94a, Yam94b] and Evain [Eva02] to study a related incidence question. See Remark 4.9 for a more detailed discussion.

Definition 2.9. For $\mathbf{c} \in \mathbb{Z}^{n}, H$ a Hilbert function, and a fixed term order $\prec$, let

$$
C_{\prec}(M)=\left\{I \in \mathbb{H}_{\mathbf{c}}(H) \mid \operatorname{in}_{\prec} I=M\right\} .
$$

This is naturally a subscheme of $\mathbb{H}_{\mathbf{c}}(H)$. Its equations can be derived from the Buchberger algorithm for computing Gröbner bases. For $M, N$ monomial ideals 
in $\mathbb{H}_{\mathbf{c}}(H)$ such that $M>N$ in the partial order of Definition 2.8 we define the edge-scheme between $M$ and $N$ to be the scheme-theoretic intersection

$$
E(M, N):=C_{\prec}(M) \cap C_{\prec \text { opp }}(N) .
$$

Altmann and Sturmfels give an algorithm to compute the edge-scheme in AS05, Algorithm 5].

Remark 2.10. In AS05] the scheme $C_{\prec}(M)$ is called the Schubert scheme $\Omega_{c}(M)$ in the case that $\mathbf{x}^{\mathbf{c}^{+}} \prec \mathbf{x}^{\mathbf{c}^{-}}$. Choosing a suitable isomorphism of $T / T^{\prime}$ with $K^{*}, C_{\prec}(M)$ consists of all points $I \in \mathbb{H}_{\mathbf{c}}(H)$ such that $\lim _{t \rightarrow 0} t \cdot I=M$. In particular, if $\mathbb{H}_{\mathbf{c}}(H)$ is smooth, $C_{\prec}(M)$ is the Białynicki-Birula cell associated to the fixed point $M$.

If $K$ is algebraically closed then $E(M, N)$ is nonempty if and only if there is an edge in the $T$-graph joining $M$ and $N$. If $K$ is not algebraically closed, the "only if" can fail, as we require the existence of a $K$-rational point $I$ in the subscheme $E(M, N)$ for there to be an edge between $M$ and $N$ in the $T$-graph. This is illustrated in the following example, which solves a problem of Altmann and Sturmfels AS05, Section $5]$.

Example 2.11. Let $S=K[x, y]$ be graded by $\mathbb{Z}^{2} / \mathbb{Z}(1,-1)$, so $\operatorname{deg}(x)=\operatorname{deg}(y)=1$. Let $M=\left\langle y^{5}, x^{2}\right\rangle$ and $N=\left\langle y^{2}, x^{5}\right\rangle$. Then the edge-scheme $E(M, N)$ is the subscheme of $\mathbb{A}^{4}$ defined by the ideal $\left\langle a^{4}-3 a^{2} b+b^{2}, c-a d, 1-b d\right\rangle$, and ideals corresponding to points in the edge-scheme are given by

$$
I=\left\langle y^{2}+a x y+b x^{2}, x^{5}\right\rangle=\left\langle y^{5}, x^{2}+c x y+d y^{2}\right\rangle
$$

This can be computed using the algorithm of [AS05, Algorithm 5], or the description given in Section 4.2. This scheme is reducible: $a^{4}-3 a^{2} b+b^{2}=\left(a^{2}-\frac{3+\sqrt{5}}{2} b\right)\left(a^{2}-\right.$ $\left.\frac{3-\sqrt{5}}{2} b\right)$. It follows from this factorization that $E(M, N)$ has $\mathbb{R}$-valued points, but no $\mathbb{Q}$-valued points. In particular, this example shows that the $T$-graph of $\operatorname{Hilb}^{10}\left(\mathbb{A}^{2}\right)$ depends on the field $K$.

2.3. Reduction to the Artinian case. In this section we prove Theorem 1.1. This is the only part of the paper to require details from [HS04]. Theorem 1.1] is only needed in this paper to apply Theorem $1.3(2)$ in the case where the Hilbert function does not have finite support, but may be of wider interest.

The ring $K$ can here be an arbitrary commutative ring; in particular $K=\mathbb{Z}$ is possible. We restrict our attention to ideals $I \subseteq S$ for which $S / I$ is a locally-free $K$ module. By the Hilbert function of a homogeneous ideal $I \subseteq S$ with $S / I$ a locally-free $K$-module, we mean the function $A \rightarrow \mathbb{N}$ given by $a \mapsto \operatorname{rk}_{K}(S / I)_{a}$.

We first recall the construction of the multigraded Hilbert scheme in the positivegraded case. The key idea is to restrict to a finite set of degrees $D$, and consider the Hilbert scheme Hilb $_{S_{D}}^{h}$, which parameterizes all locally-free $K$-modules $\oplus_{a \in D} T_{a}$ with $\operatorname{rk}_{K}\left(T_{a}\right)=h(a)$ with the property that for all $a, b \in D$ there is a multiplication map $S_{b-a} \times T_{a} \rightarrow T_{b}$. Particular examples of such $K$-modules are $\oplus_{a \in D}(S / I)_{a}$, where $I$ is an ideal with Hilbert function $h$. A major step in the construction of the multigraded Hilbert scheme is to show that for suitably chosen $D$ we have $\operatorname{Hilb}_{S_{D}}^{h} \cong \operatorname{Hilb}_{S}^{h}$. 
Recall from [HS04, Section 3] that a finite subset $D$ of the abelian group $A$ is called very supportive for a Hilbert function $h: A \rightarrow \mathbb{N}$ if it satisfies the following three conditions:

(g) Every monomial ideal with Hilbert function $h$ is generated by monomials whose degrees belong to $D$;

(h) Every monomial ideal $M$ whose generators have degrees in $D$ has the property that if $M$ has Hilbert function $h(a)$ in degree $a$ for all $a \in D$, then $M$ has Hilbert function $h$ everywhere; and

(s) For every monomial ideal $M$ with Hilbert function $h$, the syzygy module of $M$ is generated by syzygies coming from relations $x^{u} x^{v^{\prime}}=x^{v} x^{u^{\prime}}=\operatorname{lcm}\left(x^{u}, x^{v}\right)$ among generators $x^{u}, x^{v}$ of $M$ such that $\operatorname{deg}\left(\operatorname{lcm}\left(x^{u}, x^{v}\right)\right) \in D$.

Theorem 3.6 of [HS04] says if $D \subset A$ is very supportive, then $\mathrm{Hilb}_{S_{D}}^{h} \cong \mathrm{Hilb}_{S}^{h}$, and [HS04, Proposition 3.2] implies that such sets exist for any grading.

Note that for every positive grading by an abelian group $A$ there exists a group homomorphism $\phi: A \rightarrow \mathbb{Z}$ with $\phi(a)>0$ whenever $\mathrm{rk} S_{a}>0$ and $a \neq 0$.

Lemma 2.12. Suppose the A-grading of $S$ is positive, so there exists a group homomorphism $\phi: A \rightarrow \mathbb{Z}$ with $\phi(a)>0$ whenever $\operatorname{rk} S_{a}>0$ and $a \neq 0$. Let $D$ be $a$ very supportive set for $h$, and choose $N>0$ with the property that $\phi(a)<N$ for all $a \in D$. Define $\bar{h}: A \rightarrow \mathbb{N}$ by

$$
\bar{h}(a)= \begin{cases}h(a) & \text { if } \phi(a)<N, \\ 0 & \text { otherwise. }\end{cases}
$$

Let $D^{\prime}=D \cup\{a: N \leqslant \phi(a) \leqslant B(N)\}$, where every degree a of a generator or minimal syzygy of the monomial ideal $\left\langle x^{u}: \phi\left(\operatorname{deg}\left(x^{u}\right)\right) \geqslant N\right\rangle$ has $\phi(a) \leqslant B(N)$. Then $D^{\prime}$ is a very supportive set for $\bar{h}$.

Proof. Let $M$ be a monomial ideal with Hilbert function $\bar{h}$, and let $M^{\prime}$ be the ideal generated by those monomials in $M$ whose degrees are contained in $D$. Then the Hilbert function of $M$ and $M^{\prime}$ agree for degrees in $D$ by construction. Since $D$ is very supportive for $h$, and $M^{\prime}$ is generated in degrees in $D$, by property $(h)$ for $D$ the monomial ideal $M^{\prime}$ has Hilbert function $h$ everywhere. This means that $M_{a}^{\prime}=M_{a}$ when $\phi(a)<N$.

Let $M^{\prime \prime}=M^{\prime}+\left\langle x^{u}: N \leqslant \phi\left(\operatorname{deg}\left(x^{u}\right)\right) \leqslant B(N)\right\rangle$. We claim that $M^{\prime \prime}=M$, which shows that $D^{\prime}$ satisfies condition $(g)$. Indeed, since the grading is positive, $M_{a}^{\prime \prime}=M_{a}^{\prime}$, and thus $M_{a}^{\prime \prime}=M_{a}$, when $\phi(a)<N$. By the definition of $B(N)$, we have

$$
\left\langle x^{u}: N \leqslant \phi\left(\operatorname{deg}\left(x^{u}\right)\right) \leqslant B(N)\right\rangle=\left\langle x^{u}: N \leqslant \phi\left(\operatorname{deg}\left(x^{u}\right)\right)\right\rangle,
$$

so $M_{a}^{\prime \prime}=S_{a}$ when $\phi(a) \geqslant N$, and thus $M_{a}^{\prime \prime}=M_{a}$ when $\phi(a) \geqslant N$. Thus $M^{\prime \prime}=M$ as required.

Since $D$ is very supportive for $h$, all syzygies between generators of the ideal $M^{\prime}$ are in degrees in $D$. By the construction of $B(N)$, all minimal syzygies between generators of $\left\langle x^{u}: N \leqslant \phi\left(\operatorname{deg}\left(x^{u}\right)\right)\right\rangle$ have degrees in $D^{\prime}$. Finally, if $x^{u}$ is a minimal generator of $M^{\prime}$ and $x^{v}$ is a minimal generator of $M$ whose degree is not in $D$, then there is some multiple $x^{u^{\prime}}$ of $x^{u}$ that is a minimal generator of $\left\langle x^{u}: N \leqslant \phi\left(\operatorname{deg}\left(x^{u}\right)\right)\right\rangle$. The degree $a$ of the syzygy between $x^{u}$ and $x^{v}$ has $N \leqslant \phi(a) \leqslant \phi(b)$, where $b$ is degree 
of the syzygy between $x^{u^{\prime}}$ and $x^{v}$, so the fact that $a \in D^{\prime}$ follows from the fact that $b \in D^{\prime}$. Thus $M$ satisfies condition $(s)$.

Suppose now that $M$ is a monomial ideal whose generators have degrees in $D^{\prime}$, and for which the Hilbert function of $M$ agrees with $\bar{h}$ for degrees in $D^{\prime}$. As before, let $M^{\prime}$ be the ideal generated by those monomials in $M$ with degrees belonging to $D$. Since $D$ is very supportive for $h$, and $M^{\prime}$ has Hilbert function $h$ in degrees in $D, M^{\prime}$ has Hilbert function $h$, and thus $M_{a}^{\prime}=M_{a}$ when $\phi(a)<N$. Since $\bar{h}(a)=0$ whenever the $N \leqslant \phi(a) \leqslant B(N), M$ contains $\left\langle x^{u}: N \leqslant \phi\left(\operatorname{deg}\left(x^{u}\right)\right) \leqslant B(N)\right\rangle=\left\langle x^{u}\right.$ : $\left.N \leqslant \phi\left(\operatorname{deg}\left(x^{u}\right)\right)\right\rangle$, so $M_{a}=S_{a}$ when $\phi(a) \geqslant N$. Thus $M$ has Hilbert function $\bar{h}$, so condition $(h)$ is satisfied.

We can now prove Theorem 1.1.

Proof of Theorem 1.1. Fix a very supportive set $D$ for $h$. By [HS04, Theorem 3.6] we have $\operatorname{Hilb}_{S_{D}}^{h} \cong \operatorname{Hilb}_{S}^{h}$. Choose a group homomorphism $\phi: A \rightarrow \mathbb{Z}$ with $\phi(a)>0$ whenever $\operatorname{rk} S_{a}>0$ and $a \neq 0$, and choose $N>0$ with the property that $\phi(a)<N$ for all $a \in D$. Define $\bar{h}: A \rightarrow \mathbb{N}$ by setting $\bar{h}(a)=h(a)$ if $\phi(a)<N$, and $\bar{h}(a)=0$ otherwise. Let $D^{\prime}=D \cup\{a: N \leqslant \phi(a) \leqslant B(N)\}$, where $B(N)$ is as in Lemma 2.12. By Lemma 2.12, $D^{\prime}$ is a very supportive set for $\bar{h}$, so Hilb ${ }_{S}^{\bar{h}} \cong \operatorname{Hilb}_{S_{D^{\prime}}}^{\bar{h}}$.

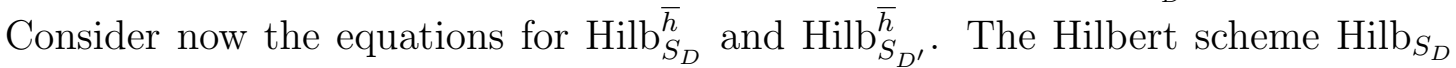
is constructed as a subscheme of the product of Grassmannians $\prod_{a \in D} G\left(h(a), S_{a}\right)$. Each ideal $I \in$ Hilb $_{S_{D}}$ gives rise to the codimension- $h(a)$ subspace $I_{a}$ of $S_{a}$. The equations defining the Hilbert scheme are the quadratic equations in the Plücker coordinates on the Grassmannians that record the fact that for $a, b \in D, x^{u} I_{a} \subseteq I_{b}$ for all $x^{u} \in S_{b-a}$. See [HS04, Corollary 3.15] for more details.

Since $\bar{h}(a)=0$ for all $a \in D^{\prime} \backslash D$, the Grassmannian $G\left(\bar{h}(a), S_{a}\right)$ is a point for all such $a$, so the second Hilbert scheme embeds into the same product of Grassmannians as the first. All quadratic equations in either case then come from pairs $a, b \in D$, so the equations defining each Hilbert scheme coincide, and Hilb ${ }_{S_{D^{\prime}}}^{\bar{h}} \cong$ Hilb $_{S_{D}}^{\bar{h}}$. Since $\bar{h}(a)=h(a)$ for all $a \in D$, we have $\operatorname{Hilb}_{S_{D}}^{\bar{h}} \cong \operatorname{Hilb}_{S_{D}}^{h}$. The choice of $D$ being very supportive means that $\operatorname{Hilb}_{S_{D}}^{h} \cong \operatorname{Hilb}_{S}^{h}$, so Hilb ${ }_{S}^{h} \cong \operatorname{Hilb}_{S}^{\bar{h}}$ as required.

Remark 2.13. Note that Theorem 1.1 implies that every pathology that exists for a positively-graded multigraded Hilbert scheme also exists for one where the Hilbert function $\bar{h}$ has finite support. These can be thought of as fixed-loci for group actions on Hilbert schemes of points in $\mathbb{A}^{n}$, so this means that all (positively-graded) Hilbert schemes are of this form. In particular, there must exist such Hilbert schemes that are disconnected (from [San05]), and that have non-reduced components (from [Mum62]).

Corollary 2.14. To decide whether there is an edge in the T-graph between a pair of monomial ideals $M, N \in$ Hilb $_{S}^{h}$, it suffices to assume that $S$ is graded by $\mathbb{Z}^{n} / \mathbf{c} \mathbb{Z}$, and $\sum_{a \in \mathbb{Z}^{n} / \mathbf{c} \mathbb{Z}} h(a)<\infty$.

Proof. By Corollary 2.6, there is an edge between $M$ and $N$ if and only if there is $\mathbf{c} \in \mathbb{Z}^{n}$ and $H: \mathbb{Z}^{n} / \mathbb{Z} \mathbf{c} \rightarrow \mathbb{N}$ for which $M, N \in \mathbb{H}_{\mathbf{c}}(H)$, and there is an edge between $M$ and $N$ in $\mathbb{H}_{\mathbf{c}}(H)$. The resulting grading by $\mathbb{Z}^{n} / \mathbb{Z} \mathbf{c}$ is positive, so by Theorem 1.1 

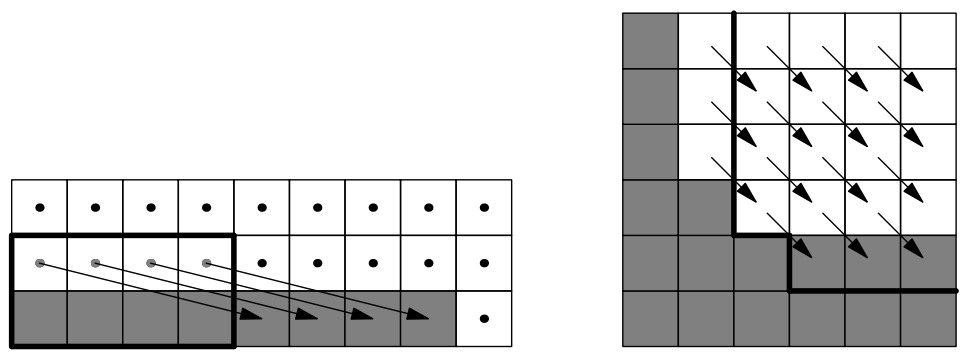

Figure 1. The arrow maps of Example 3.1.

there is $H^{\prime}$ with $\sum_{a \in \mathbb{Z}^{n} / \mathbb{Z} \mathbf{c}} H^{\prime}(a)<\infty$ and $\mathbb{H}_{\mathbf{c}}(H) \cong \mathbb{H}_{\mathbf{c}}\left(H^{\prime}\right)$. Thus there is an edge between $M$ and $N$ in $\mathbb{H}_{\mathbf{c}}(H)$ if and only if there is an edge between the ideals corresponding to $M$ and $N$ in $\mathbb{H}_{\mathbf{c}}\left(H^{\prime}\right)$.

\section{NeCESsary COnditions For a $T$-EDGE}

In this section we prove Theorem 1.3 , giving necessary conditions for the existence of an edge in the $T$-graph between two monomial ideals in $\mathbb{H}_{\mathbf{c}}(H)$. By Corollary 2.14, this gives a necessary condition for there to be an edge between monomial ideals in any $\operatorname{Hilb}_{S}^{h}$. The condition that the Hilbert function $H$ has $\sum_{a \in \mathbb{Z}^{n} / \mathbb{Z} \mathbf{c}} H(a)<\infty$ is unnecessary in the first part of this section, so we do not require it.

Recall the definition of an arrow map (Definition 1.2). We illustrate the concept of an arrow map in the following example.

Example 3.1. (1) Let $S=K[x, y]$ be graded by $\mathbb{Z}^{2} / \mathbb{Z}(2,-1)$, so $\operatorname{deg}(x)=1$, $\operatorname{deg}(y)=2$, and let $\prec$ be the lexicographic order with $x \prec y$. Let $M=\left\langle x^{8}, y\right\rangle$, and $N=\left\langle x^{4}, y^{2}\right\rangle$. Then an arrow map between $M$ and $N$ is given by the following set of pairs $(m, f(m)):\left\{\left(y, x^{4}\right),\left(x y, x^{5}\right),\left(x^{2} y, x^{6}\right),\left(x^{3} y, x^{7}\right)\right\} \cup\{(m, m)$ : $m \in \operatorname{Mon}\left(\left\langle x^{8}, x^{4} y, y^{2}\right\rangle\right)$. This is illustrated on the left of Figure 1 . The grey shaded monomials are the standard monomials of $M$, and the monomials encased by the thick black line are the standard monomials of $N$. A dot in the box corresponding to a monomial $m$ indicates that $f(m)=m$.

(2) Let $S=K[x, y]$ be graded by $\mathbb{Z}^{2} / \mathbb{Z}(1,-1)$, so $\operatorname{deg}(x)=\operatorname{deg}(y)=1$, and let $\prec$ be the lexicographic order with $x \prec y$. Let $M=\left\langle x^{2} y^{2}, x y^{3}\right\rangle$, and $N=\left\langle x^{3} y, x^{2} y^{2}\right\rangle$. Then an arrow map between $M$ and $N$ is given by setting $f\left(x^{a} y^{b}\right)=x^{a+1} y^{b-1}$ for all $x^{a} y^{b} \in M$. This is illustrated on the right of Figure 1.

Proposition 3.2. Let I be homogeneous with respect to a positive $\mathbb{Z}^{n} / \mathbb{Z} \mathbf{c}$-grading, and let $\prec$ be a term order on $S$. Set $M=\operatorname{in}_{\prec} I$, and $N=$ in $_{\prec \text { opp }} I$. The map $f: \operatorname{Mon}(M) \rightarrow \operatorname{Mon}(N)$ defined by

$$
m \mapsto \max _{\prec}\left\{\operatorname{in}_{\prec \text { opp }}(p) \mid p \in I \text { homogeneous and } \operatorname{in}_{\prec}(p)=m\right\}
$$

is an arrow map.

Proof. First note that the image of $f$ is contained in $\operatorname{Mon}(N)$, since in $\operatorname{ropp}(I)=$ $N$. Moreover, it follows from the definition that $f$ is degree-preserving and $m \succeq$ 
$f(m)$. For every $m \in \operatorname{Mon}(M)$ choose a homogeneous polynomial $p_{m} \in I$ such that $\operatorname{in}_{\prec}\left(p_{m}\right)=m$ and $\operatorname{in}_{\prec \text { opp }}\left(p_{m}\right)=f(m)$.

To see that $f$ is a bijection, we first show it is injective. Assume $f(m)=f\left(m^{\prime}\right)$ for $m \succ m^{\prime}$. We denote by $\operatorname{lc}(f)$ the leading coefficient of a polynomial $f$. This is the coefficient of the largest monomial occurring in $f$ with respect to the term order. Then $q=\mathrm{lc}_{\prec \text { opp }}\left(p_{m^{\prime}}\right) p_{m}-\mathrm{lc}_{\prec \text { opp }}\left(p_{m}\right) p_{m^{\prime}} \in I$ has $\operatorname{in}_{\prec} q=m$, and $\operatorname{in}_{\prec \text { opp }} q \succ f(m)$, which contradicts the construction of $f(m)$. Since $M$ and $N$ have the same $\mathbb{Z}^{n} / \mathbb{Z} \mathbf{c}$ graded Hilbert function, and the grading is positive, any degree preserving injection $\operatorname{Mon}(M) \rightarrow \operatorname{Mon}(N)$ is a bijection.

To see condition (2) of the definition of an arrow map, let $m \in \operatorname{Mon}(M)$ and fix a multiple $m^{\prime}=m^{\prime \prime} m$ of $m$. Note that $m^{\prime \prime} p_{m} \in I$ is homogeneous, with in ${ }_{\prec}\left(m^{\prime \prime} p_{m}\right)=$ $m^{\prime}$ and $\operatorname{in}_{\prec \text { opp }}\left(m^{\prime \prime} p_{m}\right)=m^{\prime \prime} f(m)$. Hence $f\left(m^{\prime}\right) \succeq m^{\prime \prime} f(m)$ and so $d\left(m^{\prime}, f\left(m^{\prime}\right)\right) \leqslant$ $d(m, f(m))$.

To show condition (3) holds, we use the following fact:

$(\dagger)$ If there exists a homogeneous polynomial $p \in I$ with in $\prec(p)=$ $m$, in $_{\prec \text { opp }}(p)=m^{\prime}$, then there exists $\widetilde{m} \in \operatorname{Mon}(M)$ such that $\widetilde{m} \preceq m$ and $f(\widetilde{m})=m^{\prime}$. In particular $d\left(\widetilde{m}, m^{\prime}\right) \leqslant d\left(m, m^{\prime}\right)$.

We prove this fact by induction on $d\left(m, m^{\prime}\right)$. If $d\left(m, m^{\prime}\right)=0$, then $m=m^{\prime}=\widetilde{m}=$ $f(m)$, and we can take $\widetilde{m}=m$. Otherwise, let $p_{m} \in I$ be a homogeneous polynomial with $\operatorname{in}_{\prec}\left(p_{m}\right)=m$ and $\operatorname{in}_{\prec \text { opp }}\left(p_{m}\right)=f(m)$. If $f(m)=m^{\prime}$, we are done. Assume $f(m) \neq m^{\prime}$. Then for $q=\mathrm{lc}_{\prec}\left(p_{m}\right) p-\mathrm{lc}_{\prec}(p) p_{m} \in I$, we have in $\prec$ opp $(q)=m^{\prime}$, and $m \succ$ in $\prec(q)=: m_{q}$. Since $d\left(m_{q}, m^{\prime}\right)<d\left(m, m^{\prime}\right)$, there exists $\widetilde{m} \preceq m_{q} \prec m$ with $f(\widetilde{m})=m^{\prime}$ by the induction hypothesis, finishing the proof of $(\dagger)$.

To see condition (3), let $m \in \operatorname{Mon}(N)$ and fix a multiple $m^{\prime}=m m^{\prime \prime}$ of $m$. Note that for $p=m^{\prime \prime} p_{f^{-1}(m)}$, we have $\operatorname{in}_{\prec}(p)=f^{-1}(m) m^{\prime \prime}$ and $\operatorname{in}_{\prec \text { opp }}(p)=m m^{\prime \prime}=m^{\prime}$. By $(\dagger)$ there exists $\widetilde{m} \prec f^{-1}(m) m^{\prime \prime}$ with $f(\widetilde{m})=m$, and (3) follows.

The following Corollary is a more general version of the first part of Theorem 1.3 .

Corollary 3.3. Let $M, N$ be monomial ideals in an arbitrary multigraded Hilbert scheme and assume that there exists an edge between $M$ and $N$ in the T-graph. Then there exists an arrow map $f: M \rightarrow N$ with respect to some grading by $\mathbb{Z}^{n} / \mathbb{Z} \mathbf{c}$ and some term order $\prec$.

Example 3.4. Let $K[x, y]$ have the standard grading, so $\mathbf{c}=(1,-1)$, and let $\prec$ be the lexicographic term order with $x \prec y$. Let $I=\left\langle x^{2}+2 y x+2 y^{2}, y^{4}\right\rangle$. When $\operatorname{char}(K) \neq 2, \operatorname{in}_{\prec} I=\left\langle x^{4}, y^{2}\right\rangle$ and $\operatorname{in}_{\prec \text { opp }} I=\left\langle x^{2}, y^{4}\right\rangle$. The arrow map induced by $I$ is given by $f\left(y^{2}\right)=x^{2}, f\left(y^{3}\right)=x^{2} y, f\left(x y^{2}\right)=x^{3}, f\left(x y^{3}\right)=x^{3} y$ and $f(m)=m$ for all other $m \in\left\langle x^{4}, y^{2}\right\rangle$.

Example 3.5. Let $S=K\left[x_{1}, x_{2}, x_{3}, x_{4}\right]$ be graded in by $\mathbb{Z}^{4} / \mathbb{Z}(2,-1,0,0)$, and fix $\prec$ with $x_{1} \succ x_{2} \succ x_{3} \succ x_{4}$. Let $M=\left\langle x_{1}^{2}, x_{2}^{2}, x_{3}^{2}, x_{4}^{2}, x_{1} x_{2} x_{3}, x_{1} x_{4}, x_{2} x_{4}, x_{3} x_{4}\right\rangle$, and let $N=\left\langle x_{2}, x_{1}^{4}, x_{1}^{3} x_{3}, x_{3}^{2}, x_{1} x_{4}, x_{3} x_{4}, x_{4}^{2}\right\rangle$. The ideals $M$ and $N$ have the same $\mathbb{Z}^{4} / \mathbb{Z}(2,-1,0,0)$-graded Hilbert function $H$. The Hilbert scheme $\mathbb{H}_{\mathbf{c}}(H)$ is a subscheme of $\operatorname{Hilb}^{8}\left(\mathbb{A}^{4}\right)$. The function $\operatorname{Mon}(M) \rightarrow \operatorname{Mon}(N)$ given by $f\left(x_{1}^{2}\right)=x_{2}$, $f\left(x_{1}^{3}\right)=x_{1} x_{2}, f\left(x_{1}^{2} x_{3}\right)=x_{2} x_{3}$ and $f(m)=m$ otherwise is an arrow map. This arrow map comes from the ideal $I=\left\langle x_{2}-x_{1}^{2}, x_{1}^{4}, x_{1}^{3} x_{3}, x_{3}^{2}, x_{1} x_{4}, x_{3} x_{4}, x_{4}^{2}\right\rangle$. 
While Proposition 3.2 shows that an ideal gives an arrow map, the following example shows that not all arrow maps are induced by an ideal.

Example 3.6. Let $S=K[x, y]$ be graded by $\mathbb{Z}^{2} / \mathbb{Z}(1,-1)$, so $\operatorname{deg}(x)=\operatorname{deg}(y)=1$, and let $M=\left\langle y^{5}, x^{2}\right\rangle$ and $N=\left\langle y^{2}, x^{5}\right\rangle$, as in Example 2.11.

Let $\prec$ be the lexicographic order with $x \prec y$. Any arrow map $f$ from $\operatorname{Mon}(M)$ to $\operatorname{Mon}\left(M^{\prime}\right)$ must satisfy $f\left(y^{2}\right)=x^{2}, f\left(y^{3}\right)=x^{2} y, f\left(x y^{2}\right)=x^{3}, f\left(y^{4}\right)=x^{2} y^{2}, f\left(x y^{3}\right)=$ $x^{3} y$, and $f\left(x^{2} y^{2}\right)=x^{4}$, and $f(m)=m$ if $\operatorname{deg}(m) \geqslant 6$. However there are three possibilities for the map $f$ in degree five. In all cases we have $f\left(y^{5}\right)=y^{5}$ and $f\left(x^{5}\right)=$ $x^{5}$, but we can have $\left\{f\left(x y^{4}\right)=x^{2} y^{3}, f\left(x^{2} y^{3}\right)=x^{4} y, f\left(x^{3} y^{2}\right)=x^{3} y^{2}\right\},\left\{f\left(x y^{4}\right)=\right.$ $\left.x^{3} y^{2}, f\left(x^{2} y^{3}\right)=x^{2} y^{3}, f\left(x^{3} y^{2}\right)=x^{4} y\right\}$, or $\left\{f\left(x y^{4}\right)=x^{2} y^{3}, f\left(x^{2} y^{3}\right)=x^{3} y^{2}, f\left(x^{3} y^{2}\right)=\right.$ $\left.x^{4} y\right\}$. Of these, only the last one is induced by an ideal as in the statement of Proposition 3.2. Indeed, for any ideal $I \in E(M, N)$ we have $a x y^{4}+b x^{2} y^{3} \in I$, and the equations for $E(M, N)$ imply that $b \neq 0$ and $a \neq 0$. So $f\left(x y^{4}\right)=x^{2} y^{3}$ for any arrow map induced from $I \in E(M, N)$. The analogous equation $c x^{4} y+d x^{3} y^{2} \in I$ rules out the second possibility.

Proof of Theorem 1.3. The first part of the theorem is a special case of Corollary 3.3. For the second, we first observe that for $I \in \mathbb{H}_{\mathbf{c}}(H)$ we have $Q \subseteq I$. Indeed, for every $i$ choose whichever term order $\prec$ or $\prec^{\text {opp }}$ agrees with the lexicographic term order with $x_{i}$ smallest. Since $x_{i}^{r_{i}} \in M \cap N$, there exists $f$ with initial term $x_{i}^{r_{i}}$ with respect to this term order, so $x_{i}^{r_{i}} \in I$.

To see the existence of an arrow map $\hat{f}: \operatorname{Mon}((Q: M)) \rightarrow \operatorname{Mon}(Q: N))$, it suffices to show that for any term order $\prec$ we have

$$
\operatorname{in}(Q: I)=(Q: \operatorname{in}(I)) .
$$

This means that $(Q: I)$ is homogeneous with respect to the $\mathbb{Z}^{n} / \mathbb{Z} \mathbf{c}$-grading with two initial ideals $\operatorname{in}_{\prec}(Q: I)=(Q: M)$ and $\operatorname{in}_{\prec \text { opp }}(Q: I)=(Q: N)$. The existence of an arrow map now follows from Proposition 3.2 .

For any ideals $J, K$, we have in $(J) \operatorname{in}(K) \subseteq \operatorname{in}(J K)$, so $\operatorname{in}(Q: I) \operatorname{in}(I) \subseteq \operatorname{in}((Q$ : $I) I) \subseteq$ in $Q=Q$. This implies that $\operatorname{in}(Q: I) \subseteq(Q: \operatorname{in}(I))$. Since $\operatorname{dim}_{K}(S / \operatorname{in}(Q:$ $I))=\operatorname{dim}_{K} S /(Q: I)$, to show equality it suffices to show that $\operatorname{dim}_{K}(S /(Q: I))=$ $\operatorname{dim}_{K}(S /(Q: \operatorname{in}(I)))$.

Note that $S / Q$ is a zero-dimensional ring that is a complete intersection, hence Gorenstein. Thus $D(-)=\operatorname{Hom}_{S / Q}(-, S / Q)$ is a dualizing functor from the category of finitely generated $S / Q$-modules to itself (see [Eis95, $\S 21.1$ and $\S 21.2]$ ). Since $Q \subset I$, $S / I$ is a $S / Q$-module. We have an isomorphism $D(S / I)=\operatorname{Hom}_{S / Q}(S / I, S / Q) \cong$ $(0: S / Q I / Q)=(Q: I) / Q$, where the isomorphism takes $\phi \in \operatorname{Hom}_{S / Q}(S / I, S / Q)$ to $\phi(1)$. Thus $\operatorname{dim}_{K}(S / I)=\operatorname{dim}_{K}(D(S / I))=\operatorname{dim}_{K}((Q: I) / Q)=\operatorname{dim}_{K}(S / Q)-$ $\operatorname{dim}_{K}(S /(Q: I))$. The desired equality follows from the fact that $\operatorname{dim}_{K}(S / I)=$ $\operatorname{dim}_{K}(S / \operatorname{in}(I))$.

The following example shows that the conditions of Theorem 1.3 (1) and (2) are not equivalent. In particular, for monomial ideals $M$ and $N$, the existence of an arrow map $f: M \rightarrow N$ is not sufficient for the edge $E(M, N)$ to be nonempty. We do not have an example where both conditions of Theorem 1.3 are not sufficient. 
FigurE 2. The arrow maps and systems of arrows of Example 3.7 .
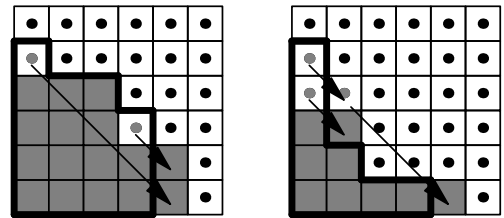

Example 3.7. Let $S=K[x, y]$ be graded by $\operatorname{deg}(x)=\operatorname{deg}(y)=1$ and let $\prec$ be the lexicographic order with $x \prec y$. Let $M=\left\langle x^{5}, x^{3} y^{2}, y^{4}\right\rangle, N=\left\langle x^{4}, x^{3} y^{3}, x y^{4}, y^{5}\right\rangle$, and let $Q=\left\langle x^{5}, y^{5}\right\rangle$. This is illustrated in Figure 2, Then the map $f: \operatorname{Mon}(M) \rightarrow$ $\operatorname{Mon}(N)$ defined by $f\left(y^{4}\right)=x^{4}, f\left(x^{3} y^{2}\right)=x^{4} y$, and $f(m)=m$ otherwise is an arrow map.

However, $(Q: M)=\left\langle x^{5}, x^{2} y, y^{3}\right\rangle$ and $(Q: N)=\left\langle x^{4}, x^{2} y, x y^{2}, y^{5}\right\rangle$. There is no arrow map $g:(Q: M) \rightarrow(Q: N)$. If there were an arrow map $g$, by (1) we would have $g\left(y^{3}\right)=x y^{2}$ and $g\left(x^{2} y\right)=x^{2} y$. Then (2) applied to $y^{3}$ implies that $g\left(y^{4}\right)=x y^{3}$ and $g\left(x y^{3}\right)=x^{2} y^{2}$, a contradiction to (3) applied to $x^{2} y$. Note however that the map given by $g\left(y^{3}\right)=x y^{2}, g\left(x^{2} y\right)=x^{2} y, g\left(y^{4}\right)=x y^{3}, g\left(x y^{3}\right)=x^{4}$ is a system of arrows in the sense of [Eva02]; see Remark 4.9. Compare [Yam94a, Section 4], [Eva02, Section $5]$.

\section{The Hilbert SCHEME OF POINTS IN THE PlAnE}

In this section we discuss in more detail the case of the Hilbert scheme $\operatorname{Hilb}^{d}\left(\mathbb{A}^{2}\right)$ of $d$ points in the plane. In this case the vertices of the $T$-graph correspond to partitions of $d$.

4.1. The basic structure of the $T$-graph of $\operatorname{Hilb}^{d}\left(\mathbb{A}^{2}\right)$. As explained in Corollary 2.6 and Remark 2.7, the $T$-graph of $\operatorname{Hilb}^{d}\left(\mathbb{A}^{2}\right)$ decomposes as a union of $T$-graphs of finitely many different $\mathbb{H}_{\mathbf{c}}(H)$ where $\mathbf{c} \in \mathbb{Z}^{2}$ with $\mathbf{c}^{+}, \mathbf{c}^{-} \neq 0$, and $H: \mathbb{Z}^{2} / \mathbb{Z} \mathbf{c} \rightarrow$ $\mathbb{N}$ is a Hilbert function. In this situation, $\mathbb{H}_{\mathbf{c}}(H)$ is smooth and irreducible; see [Eva04, Ive72, MS10]. This is not true when $S$ has more than two variables.

Up to sign we have $\mathbf{c}=(\beta,-\alpha)$, where $\alpha, \beta \in \mathbb{Z}_{>0}$ are relatively prime. Thus $S=K[x, y]$ has a $\mathbb{Z}$-grading by $\operatorname{deg}(x)=\alpha, \operatorname{deg}(y)=\beta$, and $\mathbb{H}_{\mathbf{c}}(H)$ consists of all ideals that are homogeneous with respect to this positive grading and that have Hilbert function $H$.

Recall from Definition 2.9 that for monomial ideals $M, N$ in $\mathbb{H}_{\mathbf{c}}(H)$ the edgescheme $E(M, N)$ of one-dimensional torus orbits connecting $M$ and $N$ is given by $C_{\prec}(M) \cap C_{\prec \text { opp }}(N)$. Note that $E(M, N)$ is is empty unless $M>N$ in the partial order of Definition 2.8.

For the remainder of this section, we let $\prec$ denote the lexicographic term order with $x \prec y$ and $\prec$ opp denote the lexicographic term order with $y \prec x$.

Definition 4.1. Fix a $\mathbb{Z}^{2} / \mathbb{Z} \mathbf{c}$-grading, and let $M \subset K[x, y]$ be a monomial ideal of finite length. Let $m_{0}=x^{a_{0}} \prec m_{1}=x^{a_{1}} y^{b_{1}} \ldots \prec m_{e}=y^{b_{e}}$ be the minimal generators of $M$ and let $r=x^{\beta} y^{-\alpha}$.

Let $w_{i}=\operatorname{lcm}\left(m_{i-1}, m_{i}\right)$. A positive significant arrow is a pair $c_{i}^{\ell}=\left(m_{i}, \ell\right)$, where $\ell \in \mathbb{Z}_{>0}$, such that the monomial $m_{i} r^{\ell}$ is a monomial not in $M$, and such that 
FiguRE 3. The positive significant arrows for the monomial ideal $\left\langle x^{8}, x^{5} y, x^{3} y^{3}, y^{4}\right\rangle$ of Example 4.3 . Here $c_{1}^{1}$ is green, $c_{2}^{1}, c_{2}^{3}$ are blue, and $c_{3}^{1}, c_{3}^{2}, c_{3}^{3}$ are red.

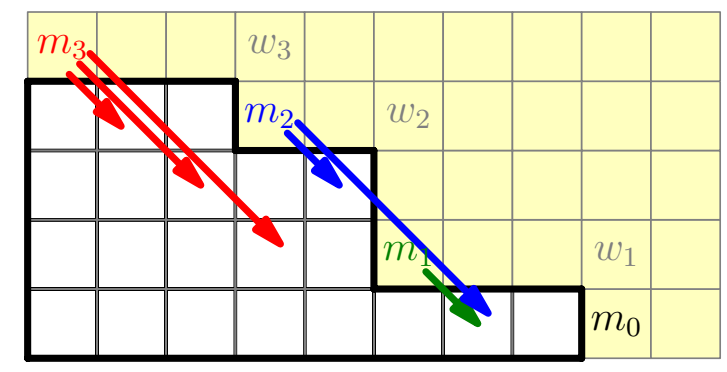

$w_{i} r^{\ell} \in M$. A negative significant arrow is a pair $c_{i}^{\ell}=\left(m_{i}, \ell\right)$, where $\ell \in \mathbb{Z}_{<0}$, such that the monomial $m_{i+1} r^{\ell}$ is a monomial not in $M$ and $w_{i+1} r^{\ell} \in M$. We denote by $T_{+}(M)$ the set of positive significant arrows, and by $T_{-}(M)$ the set of negative significant arrows. Note that $w_{i}=x^{a_{i-1}} y^{b_{i}}$, and the condition that $m_{i} r^{l}$ is a monomial means that $\ell \alpha \leqslant b_{i}$.

Definition 4.2. To every monomial $m \in M$ we associate a minimal generator $m_{j(m)}$ of $M$, where

$$
j(m)=\max \left\{j \mid m_{j} \text { divides } m\right\} .
$$

Let $f_{0}=m_{0}$ and define recursively

$$
f_{i}=\frac{m_{i}}{m_{i-1}} f_{i-1}+\sum_{c_{i}^{\ell} \in T_{+}(M)} c_{i}^{\ell} \frac{m_{i} r^{\ell}}{m_{j\left(w_{i} r^{\ell}\right)}} f_{j\left(w_{i} r^{\ell}\right)} \in K\left[T_{+}(M)\right][x, y],
$$

where we abuse notation by identifying the significant arrow $c_{i}^{\ell}$ with the corresponding variable. That $f_{i}$ is a polynomial (as opposed to merely a Laurent polynomial) follows by induction. Let

$$
I_{\prec}(M)=\left\langle f_{0}, \ldots, f_{e}\right\rangle \subset K\left[T_{+}(M)\right][x, y] .
$$

Example 4.3. Let $K[x, y]$ have the standard grading $\operatorname{deg}(x)=\operatorname{deg}(y)=1$. Then $r=x y^{-1}$. Let $M=\left\langle x^{8}, x^{5} y, x^{3} y^{3}, y^{4}\right\rangle$. Then $m_{0}=x^{8}, m_{1}=x^{5} y, m_{2}=x^{3} y^{3}$ and $m_{3}=y^{4}$, and we have $w_{1}=x^{8} y, w_{2}=x^{5} y^{3}$, and $w_{3}=x^{3} y^{4}$. The positive significant arrows are $T_{+}(M)=\left\{c_{1}^{1}, c_{2}^{1}, c_{2}^{3}, c_{3}^{1}, c_{3}^{2}, c_{3}^{3}\right\}$. This is illustrated in Figure 3 . The polynomials are

$$
\begin{aligned}
f_{0}= & x^{8} \\
f_{1}= & x^{5} y+c_{1}^{1} x^{6} \\
f_{2}= & x^{3} y^{3}+\left(c_{1}^{1}+c_{2}^{1}\right) x^{4} y^{2}+\left(c_{2}^{1} c_{1}^{1}\right) x^{5} y+c_{2}^{3} x^{6} \\
f_{3}= & y^{4}+\left(c_{1}^{1}+c_{2}^{1}+c_{3}^{1}\right) x y^{3}+\left(c_{2}^{1} c_{1}^{1}+c_{3}^{1} c_{1}^{1}+c_{3}^{1} c_{2}^{1}+c_{3}^{2}\right) x^{2} y^{2} \\
& +\left(c_{2}^{3}+c_{3}^{1} c_{2}^{1} c_{1}^{1}+c_{3}^{2} c_{1}^{1}+c_{3}^{3}\right) x^{3} y+\left(c_{3}^{1} c_{2}^{3}+c_{3}^{3} c_{1}^{1}\right) x^{4} .
\end{aligned}
$$


Remark 4.4. The set of significant arrows of $M$, for all possible (not necessarily positive) gradings $\mathbb{Z}^{2} / \mathbb{Z} \mathbf{c}$, is in bijection with the weights of the torus action on the tangent space $T_{M} \operatorname{Hilb}^{d}\left(\mathbb{A}^{2}\right)$ to $M$ induced by the action of $T$ on $\operatorname{Hilb}^{d}\left(\mathbb{A}^{2}\right)$. The map that associates to a significant arrow $c_{i}^{\ell}$ for the grading $\mathbb{Z} / \mathbb{Z} \mathbf{c}$ the weight $\ell \mathbf{c}$ is a bijection. See for example [Nak99, Proposition 5.7], [Hai98, Proposition 2.4], or Eva04, §2]. In particular, the gradings by $\mathbb{Z}^{n} / \mathbb{Z} \mathbf{c}$ for which $\mathbb{H}_{\mathbf{c}}(H)$ is not simply the point $\{M\}$, where $H$ is the Hilbert function of $M$, are those for which there exists some significant arrow for $M$; compare Remark 2.7.

In [Eva04, Section 3], Evain gives the following parametrization of the cells $C_{\prec}(M)$ which we will use to compute equations for the edge-scheme $E(M, N)$.

Theorem 4.5. We have

(1) The set $\left\{f_{0}, \ldots, f_{e}\right\}$ is a Gröbner basis of $I_{\prec}(M)$ with respect to $\prec$ (Eva04, Proposition 10]).

(2) The map $\mathbb{A}^{\left|T_{+}(M)\right|} \rightarrow \mathbb{H}_{\mathbf{c}}(H)$ induced by $I_{\prec}(M)$ is an isomorphism onto the affine cell $C_{\prec}(M)$ (Eva04, Theorem 11]).

In the following proposition we use arguments from Evain [Eva04] to imply that $\mathbb{H}_{\mathbf{c}}(H)$ is the closure of an edge-scheme.

Proposition 4.6. Let $S=K[x, y]$ be graded by $\mathbb{Z}^{2} / \mathbb{Z} \mathbf{c}$, where $\mathbf{c} \in \mathbb{Z}^{2}$ with $\mathbf{c}^{+}, \mathbf{c}^{-} \neq 0$. Fix a Hilbert function $H$. Then there exists a unique maximal element $M_{\max }$ and a unique minimal element $M_{\min }$ with respect to the partial order of Definition 2.8 for the monomial ideals contained in $\mathbb{H}_{\mathbf{c}}(H)$, and $\mathbb{H}_{\mathbf{c}}(H)$ is the closure of the edge-scheme $E\left(M_{\max }, M_{\min }\right)$.

Proof. Evain [Eva04, Theorem 19] shows that the poset of Definition 2.8 on the monomial ideals contained in $\mathbb{H}_{\mathbf{c}}(H)$ has a unique minimal element $M_{\min }$ such that $T_{+}\left(M_{\min }\right)=\emptyset$. By Remark 4.4, the number of significant arrows at $M$ equals the dimension of the tangent space to $\mathbb{H}_{\mathbf{c}}(H)$ at $M$, which equals the dimension since $\mathbb{H}_{\mathbf{c}}(H)$ is smooth. So since $T_{+}\left(M_{\min }\right)=\emptyset$, we have $\left|T_{-}\left(M_{\min }\right)\right|=\operatorname{dim} \mathbb{H}_{\mathbf{c}}(H)$.

By switching $x$ and $y$ we switch the roles of $\prec$ and $\prec^{\text {opp }}$, and of positive and negative signficant arrows. Thus Theorem 4.5(2) also applies to $\prec$ opp and so we have $\operatorname{dim} C_{\prec \text { opp }}\left(M_{\min }\right)=\operatorname{dim} \mathbb{H}_{\mathbf{c}}(H)$.

The cells $C_{\prec}(M)$ are locally closed, so $C_{\prec \text { opp }}\left(M_{\min }\right)$ is open; see [BBCM02, Theorem 4.2]. Since $\mathbb{H}_{\mathbf{c}}(H)$ is irreducible [Eva04], it follows that $C_{\prec \text { opp }}\left(M_{\min }\right)$ is an open dense subset of $\mathbb{H}_{\mathbf{c}}(H)$. Similarly, we obtain a maximal element $M_{\max }$ such that $C_{\prec}\left(M_{\max }\right)$ is an open dense subset of $\mathbb{H}_{\mathbf{c}}(H)$. Hence the intersection $C_{\prec}\left(M_{\max }\right) \cap C_{\prec \text { opp }}\left(M_{\min }\right)$ is an open dense subset of $\mathbb{H}_{\mathbf{c}}(H)$, which implies the claim.

Remark 4.7. Our proof uses the smoothness of $\mathbb{H}_{\mathbf{c}}(H)$ in this setting, and it would be interesting to know if this closure property is true in more than two variables, where the smoothness may fail. It also suggests studying the restricted graph of just edges whose closure is the entire $\mathbb{H}_{\mathbf{c}}(H)$.

Remark 4.8. In the standard grading $\operatorname{deg}(x)=\operatorname{deg}(y)=1$, Macaulay's theorem asserts that the maximal element of the poset is the lex-segment ideal with Hilbert function $H$, which is the monomial ideal containing the $d+1-H(d)$ largest elements 
of degree $d$ for every $d$; see, for example, [BH93, Theorem 4.2.10]. This is not true for more general gradings. However, Evain gives a recursive construction of $M$ in [Eva04, Remark 23]; see also [MS10, Proposition 3.12].

Remark 4.9. Yaméogo Yam94a, Yam94b] and Evain [Eva02] studied a related incidence question in the case of $\operatorname{Hilb}^{d}\left(\mathbb{A}^{2}\right)$. In [Yam94a] Yaméogo shows that the closure of a cell $C_{\prec}(M)$ need not be a union of cells. Given two cells $C_{\prec}(M), C_{\prec}\left(M^{\prime}\right)$, one may ask whether $C_{\prec}\left(M^{\prime}\right) \subset \overline{C_{\prec}(M)}$ (strong incidence) or $C_{\prec}\left(M^{\prime}\right) \cap \overline{C_{\prec}(M)} \neq \emptyset$ (weak incidence), and in [Yam94b] Yaméogo gives a sufficient condition for strong incidence. In [Yam94a] he shows that being related in the partial order of Definition 2.8 is a necessary, yet not sufficient, condition for weak incidence. Evain strengthens this condition in [Eva02] as follows. A system of arrows is a monomial $\operatorname{map} f: \operatorname{Mon}(M) \rightarrow \operatorname{Mon}(N)$ satisfying conditions 1 and 3 in Definition 1.2 ; compare [Eva02, Definition-Proposition 11]. Evain's systems of arrows [Eva02, Definition 4] are defined to be maps on the partitions, so they go from monomials not in $M$ to monomials not in $N$. However, Evain's system of arrows is equivalent to a system of arrows in this sense from $\operatorname{Mon}(Q: M)$ to $\operatorname{Mon}(Q: N)$.

In [Eva02, Theorem 8], Evain proves that if $C_{\prec}(N) \cap \overline{C_{\prec}(M)} \neq \emptyset$ and $Q$ is as in Theorem 1.3, then there exists a system of arrows $\operatorname{Mon}(M) \rightarrow \operatorname{Mon}(N)$ and a system of arrows $\operatorname{Mon}(Q: M) \rightarrow \operatorname{Mon}(Q: N)$. No example is known where this condition is not sufficient. Note that in Example 3.7, the map given by $g\left(y^{3}\right)=x y^{2}, g\left(x^{2} y\right)=$ $x^{2} y, g\left(y^{4}\right)=x y^{3}, g\left(x y^{3}\right)=x^{4}$ is a system of arrows between $(Q: M)$ and $(Q: N)$, but there is no arrow map.

The existence of a one-dimensional torus orbit between $M$ and $N$ implies that $N \in \overline{C_{\prec}(M)}$ and $M \in \overline{C_{\prec \text { opp }}(N)}$, so Evain's theorem [Eva02, Theorem 8] implies the existence of a system of arrows from $M \rightarrow N$ and $(Q: M) \rightarrow(Q: N)$ with respect to $\prec$ as well as $\prec^{\text {opp }}$.

4.2. A combinatorial description of the edge ideal. In this section we prove Theorem 1.4 by giving an explicit combinatorial description of the equations for the edge scheme $E(M, N)$ over $\mathbb{Z}$. The algorithm in [AS05, Algorithm 5] for computing this edge works by finding Gröbner bases for $I_{\prec}(M)$ and $I_{\prec \text { opp }}(N)$ and reducing the Gröbner basis for $I_{\prec \text { opp }}(N)$ modulo the Gröbner basis $I_{\prec}(M)$. We now apply this algorithm to Evain's Gröbner basis to combinatorially describe equations for $E(M, N)$, which involves the following combinatorial constructions.

For this section we fix a $\mathbb{Z}^{2} / \mathbb{Z} \mathbf{c}$-grading, and a monomial ideal $M \subset K[x, y]$. The following definition, which uses the notation of Definition 4.1, defines the combinatorial objects we will use to give equations for the $E(M, N)$.

Definition 4.10. (1) A path from a generator $m_{i}$ of $M$ is a sequence of arrows $\mathcal{P}=\left(c_{i_{1}}^{\ell_{1}}, \ldots, c_{i_{d}}^{\ell_{d}}\right)$ where $c_{i_{k}}^{\ell_{k}} \in T_{+}(M)$, defined inductively as follows:

(a) If $c_{i}^{\ell} \in T_{+}(M)$, then $\left(c_{i}^{\ell}\right)$ is a path from $m_{i}$.

(b) Otherwise, either

(†) $\left(c_{i_{1}}^{\ell_{1}}, \ldots, c_{i_{d}}^{\ell_{d}}\right)$ is a path from $m_{i-1}$, or

$(\ddagger) i_{1}=i$, and $\left(c_{i_{2}}^{\ell_{2}}, \ldots, c_{i_{d}}^{\ell_{d}}\right)$ is a path from $m_{j\left(w_{1} r^{\ell}\right)}$.

We define the length of the path $\mathcal{P}$ to be $\ell(\mathcal{P})=\ell_{1}+\cdots+\ell_{d}$, and we say that $\mathcal{P}$ is a path from from $m_{i}$ to $m_{i} r^{\ell(\mathcal{P})}$. The construction of a path guarantees 
that $m_{i} r^{\ell(\mathcal{P})}$ is a monomial; when $d=1$ this is part of the definition of a significant arrow.

(2) A walk from a generator $m_{i}$ of $M$ to a monomial $s$ is defined to be a sequence of paths $\left(\mathcal{P}_{1}, \ldots, \mathcal{P}_{d}\right)$, such that $\mathcal{P}_{1}$ is a path from $m_{i}, \mathcal{P}_{k}$ is a path from $m_{j\left(m_{i} r^{\ell\left(\mathcal{P}_{1}\right)+\cdots \ell\left(\mathcal{P}_{k-1}\right)}\right)}$ for $2 \leqslant k \leqslant d, m_{i} r^{\ell\left(\mathcal{P}_{1}\right)+\cdots+\ell\left(\mathcal{P}_{k}\right)} \in M$ for $1 \leqslant k \leqslant d-1$, and $m_{i} r^{\ell\left(\mathcal{P}_{1}\right)+\cdots+\ell\left(\mathcal{P}_{d}\right)}=s$. We define the length of the walk to be $\ell(\mathcal{W})=$ $\ell\left(\mathcal{P}_{1}\right)+\cdots+\ell\left(\mathcal{P}_{d}\right)$.

(3) A stroll from a monomial $m \in M$ to a standard monomial $s$ is a sequence of walks $\mathcal{S}=\left(\mathcal{W}_{1}, \ldots, \mathcal{W}_{d}\right)$, such that $\mathcal{W}_{1}$ is a walk from $m_{j(m)}, \mathcal{W}_{k}$ is a walk from $m_{j\left(m r^{\ell\left(\mathcal{W}_{1}\right)+\cdots+\ell\left(\mathcal{W}_{k-1}\right)}\right)}$ for $2 \leqslant k \leqslant d, m r^{\ell\left(\mathcal{W}_{1}\right)+\cdots+\ell\left(\mathcal{W}_{k-1}\right)} \in M$, and $m r^{\ell\left(\mathcal{W}_{1}\right)+\cdots+\ell\left(\mathcal{W}_{d}\right)}=s$. There is also the trivial stroll from a standard monomial $s$ for $M$ to itself.

(4) Let $H$ be a Hilbert function, and let $M, N$ be monomial ideals in $\mathbb{H}_{\mathbf{c}}(H)$ such that $M>N$ in the partial order of Definition 2.8. A hike $\mathcal{H}$ from a minimal generator $n$ of $N$ to a standard monomial $s$ for $M$ is a pair $\left(\mathcal{P}^{\prime}, \mathcal{S}\right)$, where $\mathcal{P}^{\prime}$

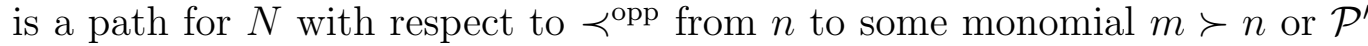
is an arrow of length zero, in which case we set $m=n$, and $\mathcal{S}$ is a stroll from $m$ to $s$.

For a path $\mathcal{P}$, we set $a_{\mathcal{P}}=\prod_{c_{i}^{\ell} \in \mathcal{P}} c_{i}^{\ell} \in K\left[T_{+}(M)\right]$. For a walk $\mathcal{W}$, we set $a_{\mathcal{W}}=$ $(-1)^{|\mathcal{W}|+1} \prod_{\mathcal{P} \in \mathcal{W}} a_{\mathcal{P}}$. For a stroll $\mathcal{S}$ we let $a_{\mathcal{S}}=\prod_{\mathcal{W} \in \mathcal{S}}(-1)^{|\mathcal{S}|} a_{\mathcal{W}}$ and for the trivial stroll $\mathcal{S}$ we let $a_{\mathcal{S}}=1$. For a hike $\mathcal{H}=\left(\mathcal{P}^{\prime}, \mathcal{S}\right)$, we let $a_{\mathcal{H}}=a_{\mathcal{P}^{\prime}} a_{\mathcal{S}}$, and if $\mathcal{P}^{\prime}$ is the arrow of length zero we set $a_{\mathcal{P}^{\prime}}=1$. Note that then $a_{\mathcal{P}}, a_{\mathcal{W}}, a_{\mathcal{S}} \in K\left[T_{+}(M)\right]$, while $a_{\mathcal{H}} \in K\left[T_{+}(M), T_{-}(N)\right]$.

We will see in the lemmas below that the notion of a path naturally comes from the recursive definition of the $f_{i}$ from Definition 4.2 , the notion of a walk from computing the reduced Gröbner basis for $I_{\prec}(M)$, and the notion of a stroll from reducing a monomial with respect to the reduced Gröbner basis.

Example 4.11. We illustrate the concepts of paths, walks, and strolls on the ideal $M=\left\langle x^{8}, x^{5} y, x^{3} y^{3}, y^{4}\right\rangle$ of Example 4.3. This is shown in Figure 4. It is convenient to describe paths, walks, strolls, and hikes as terms of polynomials in $K\left[T_{+}(M)\right]$, where the path $\mathcal{P}$ is represented by the term $a_{\mathcal{P}}$. There are no paths from $m_{0}$. The only path from $m_{1}$ is $c_{1}^{1}$, the paths from $m_{2}$ are the terms of $c_{2}^{1}+c_{1}^{1}+c_{2}^{1} c_{1}^{1}+c_{2}^{3}$, and the paths from $m_{3}$ are the terms of $c_{3}^{1}+c_{1}^{1}+c_{2}^{1}+c_{3}^{2}+c_{2}^{1} c_{1}^{1}+c_{3}^{1} c_{1}^{1}+c_{3}^{1} c_{2}^{1}+c_{3}^{3}+c_{2}^{3}+c_{3}^{1} c_{2}^{1} c_{1}^{1}+c_{3}^{2} c_{1}^{1}+c_{3}^{1} c_{2}^{3}+c_{3}^{3} c_{1}^{1}$. For $i=0,1,3$, a walk from $m_{i}$ is simply a path from $m_{i}$. The walks from $m_{2}$ are the paths from $m_{2}$ as well as $\left(c_{2}^{1} c_{1}^{1}, c_{1}^{1}\right)$.

The only stroll from $x^{5} y$ to $x^{6}$ is $c_{1}^{1}$. There is only the trivial stroll from $x^{4} y^{2}$. The strolls from $x^{3} y^{3}$ to $x^{6}$ are $c_{2}^{3}$, and $c_{2}^{1} c_{1}^{1} c_{1}^{1}$. For the more complicated strolls it is convenient to define $W_{m}^{\ell}$ to be the polynomial whose terms are walks from $j(m)$ of length $\ell$ (the $\ell$-homogeneous part of the walk polynomials computed above), and to define $S_{m, s}$ to be the polynomial whose terms are strolls from $m$ to $s$. Then $S_{x^{2} y^{4}, x^{6}}=W_{x^{2} y^{4}}^{4}+W_{x^{2} y^{4}}^{3} S_{x^{5} y, x^{6}}+W_{x^{2} y^{4}}^{1} S_{x^{3} y^{3}, x^{6}}=2 c_{3}^{1} c_{2}^{3}+c_{3}^{3} c_{1}^{1}+2 c_{2}^{3} c_{1}^{1}+2 c_{3}^{1} c_{2}^{1}\left(c_{1}^{1}\right)^{2}+$ 
FiguRe 4. The upper left picture shows the path $c_{2}^{1} c_{1}^{1}$ from $m_{2}$ and the paths $c_{3}^{3} c_{1}^{1}$ and $c_{3}^{1} c_{2}^{1} c_{1}^{1}$ from $m_{3}$; the upper right picture shows the walk $c_{2}^{1} c_{1}^{1} c_{1}^{1}$ from $m_{2}$; the lower left the stroll $c_{3}^{1} c_{2}^{1} c_{2}^{1} c_{1}^{1} c_{1}^{1}$ from $x y^{5}$; the lower right the hike $\widetilde{c}_{1}^{4} c_{3}^{1} c_{2}^{1} c_{2}^{1} c_{1}^{1} c_{1}^{1}$ from $x^{5} y$ to $x^{6}$.
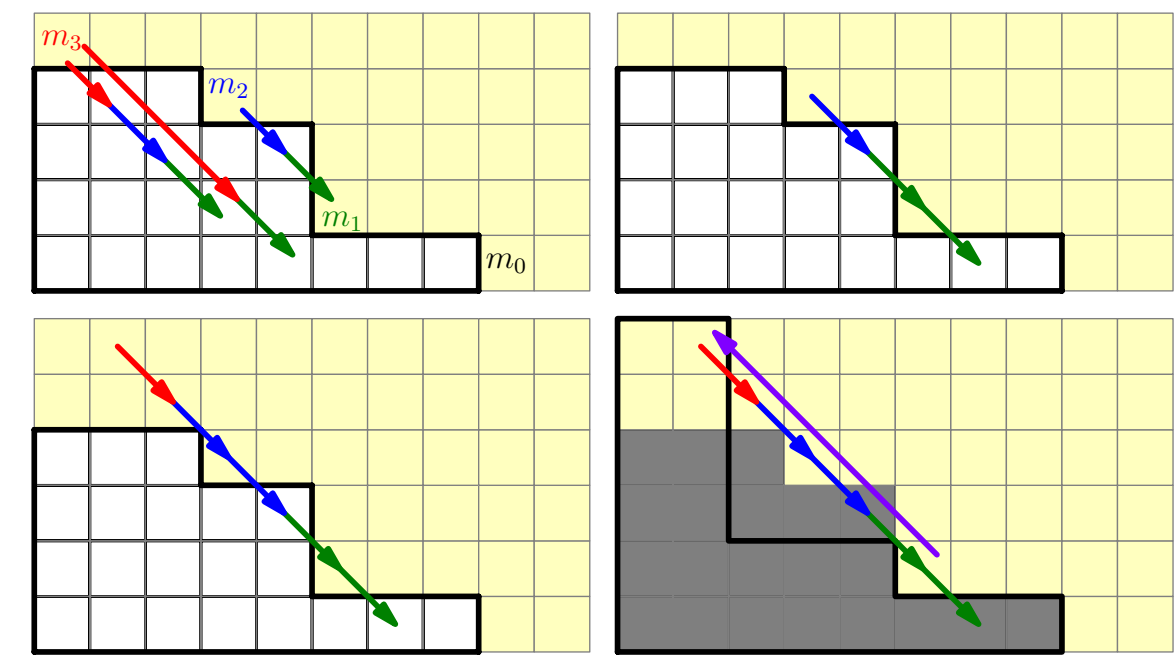

$$
\begin{aligned}
& c_{3}^{2}\left(c_{1}^{1}\right)^{2}+c_{2}^{1} c_{2}^{3}+c_{2}^{1}\left(c_{1}^{1}\right)^{3}+\left(c_{2}^{1}\right)^{2}\left(c_{1}^{1}\right)^{2} \text { and } \\
& S_{x y^{5}, x^{6}}= W_{x y^{5}}^{4} S_{x^{5} y, x^{6}}+W_{x y^{5}}^{2} S_{x^{3} y^{3}, x^{6}}+W_{x y^{5}}^{1} S_{x^{2} y^{4}, x^{6}} \\
&= 6 c_{3}^{1} c_{2}^{3} c_{1}^{1}+c_{3}^{2} c_{2}^{3}+3 c_{2}^{3} c_{2}^{1} c_{1}^{1}+4 c_{3}^{1} c_{2}^{1} c_{2}^{3}+2 c_{3}^{2} c_{2}^{1}\left(c_{1}^{1}\right)^{2} \\
&+3\left(c_{2}^{1}\right)^{2}\left(c_{1}^{1}\right)^{3}+4 c_{3}^{1} c_{2}^{1}\left(c_{1}^{1}\right)^{3}+2 c_{3}^{1}\left(c_{2}^{1}\right)^{2}\left(c_{1}^{1}\right)^{2}+2\left(c_{3}^{1}\right)^{2} c_{2}^{3} \\
&+c_{3}^{1} c_{3}^{3} c_{1}^{1}+2\left(c_{3}^{1}\right)^{2} c_{2}^{1}\left(c_{1}^{1}\right)^{2}+c_{3}^{1} c_{3}^{2}\left(c_{1}^{1}\right)^{2}+2 c_{2}^{1} c_{3}^{3} c_{1}^{1} \\
&+2 c_{3}^{1}\left(c_{2}^{1}\right)^{2}\left(c_{1}^{1}\right)^{2}+\left(c_{2}^{1}\right)^{2} c_{2}^{3}+\left(c_{2}^{1}\right)^{3}\left(c_{1}^{1}\right)^{2}+c_{3}^{3}\left(c_{1}^{1}\right)^{2} \\
&+2 c_{2}^{3}\left(c_{1}^{1}\right)^{2}+c_{3}^{2}\left(c_{1}^{1}\right)^{3}+c_{2}^{1}\left(c_{1}^{1}\right)^{4} .
\end{aligned}
$$

Let

$$
\mathcal{M}_{i}=\left\{m \text { a monomial } \mid m \prec m_{i} \text { and } \operatorname{deg}(m)=\operatorname{deg}\left(m_{i}\right)\right\},
$$

where $m_{i}$ is as in Definition 4.1 .

Lemma 4.12. We have

$$
f_{i}=m_{i}+\sum_{m \in \mathcal{M}_{i}}\left(\sum_{\substack{\mathcal{P} \text { path from } \\ m_{i} \text { to } m}} a_{\mathcal{P}}\right) m .
$$

Proof. We proceed by induction on $i$. For $i=0$, we have $\mathcal{M}_{i}=\emptyset$ and $f_{0}=m_{0}$. The coefficient of $m$ in $f_{i}$ is the coefficient of $\frac{m_{i-1}}{m_{i}} m$ in $f_{i-1}$ plus the sum over all significant positive arrows $c_{i}^{\ell}$ originating at $m_{i}$ of $c_{i}^{\ell}$ times the coefficient of $\frac{m_{j\left(w_{i} r^{\ell}\right)}}{m_{i} r^{\ell}} m$ in $f_{j\left(w_{i} r e\right)}$. By induction, the former corresponds to the paths of the form b) $(\dagger)$, and the latter to the paths of the form $b)(\ddagger)$ and a). 
Lemma 4.13. Let $\mathcal{T}_{i}=\left\{s \in \mathcal{M}_{i} \mid s \notin M\right\}$ and

$$
g_{i}=m_{i}+\sum_{s \in \mathcal{T}_{i}}\left(\sum_{\substack{\mathcal{W} \text { walk from } \\ m_{i} \text { to } s}} a_{\mathcal{W}}\right) s .
$$

Then $\left\{g_{0}, \ldots, g_{e}\right\}$ is a reduced Gröbner basis for the ideal $I_{\prec}(M)$ of Definition 4.2.

Proof. We first prove that each $g_{i}$ lies in the ideal $\left\langle f_{0}, \ldots, f_{e}\right\rangle$.

Let $\mathcal{M}_{i} \backslash \mathcal{T}_{i}=\left\{u_{1} \ldots, u_{t}\right\}$, with $u_{1} \succ \ldots \succ u_{t}$. We let $U_{k}=\left\{u_{1}, \ldots, u_{k}\right\}$ and $U_{0}=\emptyset$. A walk $\mathcal{W}=\left(\mathcal{P}_{1}, \ldots, \mathcal{P}_{d}\right)$ is a called a $k$-walk if $m_{i} r^{\ell\left(\mathcal{P}_{1}\right)+\cdots+\ell\left(\mathcal{P}_{j}\right)} \in U_{k}$ for $1 \leqslant j \leqslant d-1$. Note that we do not require $m_{i} r^{\ell(\mathcal{W})}$ to be contained in $U_{k}$. A 0 -walk is a path, and a $t$-walk is a walk.

For $0 \leqslant k \leqslant t$ we let

$$
h_{k}=m_{i}+\sum_{m \in \mathcal{M}_{i} \backslash U_{k}}\left(\sum_{\substack{\mathcal{W} \text { k-walk from } \\ m_{i} \text { to } m}} a_{\mathcal{W}}\right) m,
$$

where the inner sum is over all $k$-walks from $m_{i}$ to $m$. Note that $h_{0}=f_{i}$ and $h_{t}=g_{i}$.

Now for $k \geqslant 1$, we have

$$
\begin{aligned}
& h_{k-1}-f_{j\left(u_{k}\right)} \frac{u_{k}}{m_{j\left(u_{k}\right)}}\left(\sum_{\substack{\mathcal{W}(k-1) \text {-walk } \\
\text { from } m_{i} \text { to } u_{k}}} a_{\mathcal{W}}\right) \\
& =m_{i}+\sum_{m \in \mathcal{M}_{i} \backslash U_{k-1}}\left(\sum_{\substack{\mathcal{W}(k-1) \text {-walk } \\
\text { from } m_{i} \text { to } m}} a_{\mathcal{W}}\right) m \\
& -\left(m_{j\left(u_{k}\right)}+\sum_{\tilde{m} \in \mathcal{M}_{j\left(u_{k}\right)}}\left(\sum_{\substack{\mathcal{P} \text { path from } \\
m_{j\left(u_{k}\right)} \text { to } \tilde{m}}} a_{\mathcal{P}}\right) \widetilde{m}\right) \frac{u_{k}}{m_{j\left(u_{k}\right)}}\left(\sum_{\substack{\mathcal{W}(k-1) \text {-walk } \\
\text { from } m_{i} \text { to } u_{k}}} a_{\mathcal{W}}\right)
\end{aligned}
$$

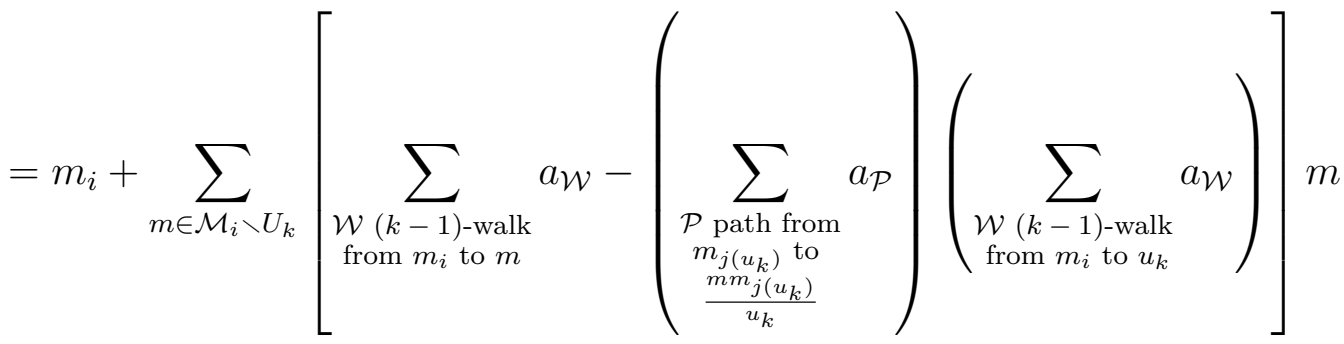

$$
\begin{aligned}
& =h_{k},
\end{aligned}
$$

where the second-to-last equality follows from the fact that the coefficient of $u_{k}$ cancels, and the last follows from the definition of a walk. It follows that $g_{i}=$ $h_{t} \in\left\langle f_{0}, \cdots, f_{e}\right\rangle$. Since $\left\langle\operatorname{in}_{\prec}\left(g_{0}\right), \ldots, \operatorname{in}_{\prec}\left(g_{e}\right)\right\rangle=M=\left\langle\operatorname{in}_{\prec}\left(f_{0}\right), \ldots, \operatorname{in}_{\prec}\left(f_{e}\right)\right\rangle$ and $\left\{f_{0}, \ldots, f_{e}\right\}$ is a Gröbner basis for $I_{\prec}(M)$ by Theorem 4.5 , it follows that $\left\{g_{0}, \ldots, g_{e}\right\}$ 
is a Gröbner basis for $I_{\prec}(M)$. The only monomials occurring in $g_{i}$ are $m_{i}$ and standard monomials, so $\left\{g_{0}, \ldots, g_{e}\right\}$ is a reduced Gröbner basis.

Lemma 4.14. Fix $m \in M$. Let $\mathcal{T}_{m}$ denote the set of monomials $s \notin M$ of the same degree as $m$ with $s \prec m$. Then

$$
m \equiv \sum_{s \in \mathcal{T}_{m}}\left(\sum_{\substack{\mathcal{S} \text { stroll from } \\ m \text { to } s}} a_{\mathcal{S}}\right) s \quad \bmod I_{\prec}(M) .
$$

Proof. We proceed by induction on the number of monomials $m^{\prime} \in M$ with $\operatorname{deg}\left(m^{\prime}\right)=$ $\operatorname{deg}(m)$ and $m^{\prime} \preceq m$. The base case is when $m$ is the smallest monomial of its degree in $M$ with respect to $\prec$. Note that in this case all monomials occurring in $\frac{m}{m_{j(m)}} g_{j(m)}$ other than $m$ are standard monomials, so we have

$$
m-g_{j(m)} \frac{m}{m_{j(m)}}=-\sum_{s=m m^{\prime} / m_{j(m)}, m^{\prime} \in \mathcal{T}_{m_{j(m)}}}\left(\sum_{\substack{\mathcal{W} \text { walk from } \\ m_{j(m)} \text { to } m^{\prime}}} a_{\mathcal{W}}\right) s .
$$

Note that since $m$ is the smallest monomial of its degree in $M$, a stroll from $m$ to a standard monomial $s$ is the same as a walk, and the base case follows. Now suppose that the claim is true whenever there are fewer smaller monomials in $M$ of the same degree. Then

$$
m-g_{j(m)} \frac{m}{m_{j(m)}}=-\sum_{m^{\prime}=\frac{m}{m_{j(m)}}}\left(\sum_{m^{\prime \prime}, m^{\prime \prime} \in \mathcal{T}_{m_{j(m)}}} a_{\substack{\mathcal{W} \text { walk from } \\ m_{j(m)} \text { to } m^{\prime} \frac{m_{j(m)}}{m}}} m^{\prime},\right.
$$

where $m^{\prime} \prec m$. If $m^{\prime}$ is standard, then a walk from $m$ to $m^{\prime}$ is a stroll. If $m^{\prime} \in M$, by induction we have

$$
m^{\prime} \equiv \sum_{s \in \mathcal{T}_{m^{\prime}}}\left(\sum_{\substack{\mathcal{S} \text { stroll from } \\ m^{\prime} \text { to } s}} a_{\mathcal{S}}\right) s \quad \bmod I_{\prec}(M) .
$$

Now a walk from $m_{j(m)}$ to $m^{\prime} \frac{m_{j(m)}}{m}$ occurring in (2) combines with a stroll from $m^{\prime}$ to $s$ occurring in (3) to give a stroll from $m$ to $s$. As every stroll occurs in this way, the claim follows.

Theorem 4.15. Fix a $\mathbb{Z}^{2} / \mathbb{Z} \mathbf{c}$-grading and a term order $\prec$ on $K[x, y]$. Let $M, N \subset$ $K[x, y]$ be monomial ideals with Hilbert function $H$. Suppose $M>N$ with respect to the partial order of Definition 2.8 induced by $\prec$. For a minimal generator $n$ of $N$, and $s$ a standard monomial for $M$ with $\operatorname{deg}(m)=\operatorname{deg}(s)$, let

$$
F_{(n, s)}=\sum_{\mathcal{H} \text { hike from n to } s} a_{\mathcal{H}} \in K\left[T_{+}(M), T_{-}(N)\right]
$$


Then the ideal for the edge-scheme $E(M, N)$ parameterizing one-dimensional torus orbits containing $M$ and $N$ is given by

$$
\left.I(M, N)=\left\langle F_{(n, s)}\right| n \text { a minimal generator of } N, s \notin M, \operatorname{deg}(n)=\operatorname{deg}(s)\right\rangle \text {. }
$$

Proof. The theorem follows from applying the ideas of [AS05, Algorithm 5] to the reduced Gröbner basis $\left\{g_{i}\right\}$ for $I_{\prec}(M)$ of Lemma 4.13 and using Lemma 4.14 to reduce the Gröbner basis $\left\{\widetilde{f}_{i}\right\}$ for $I_{\prec \text { opp }}(N)$.

The edge scheme $E(M, N)$ is the scheme-theoretic intersection $C_{\prec}(M) \cap C_{\prec \text { opp }}(N)$. This equals the fiber product $C_{\prec}(M) \times_{\mathbb{H}_{\mathbf{c}}(H)} C_{\prec \text { opp }}(N)$. Thus to show that $E(M, N)=$ $\operatorname{Spec}\left(K\left[T_{+}(M), T_{-}(N)\right] / I(M, N)\right)$, it suffices to show that the subscheme $C^{\prime}$ of $\operatorname{Spec}\left(K\left[T_{+}(M), T_{-}(N)\right]\right)=C_{\prec}(M) \times C_{\prec \text { opp }}(N)$ defined by $I(M, N)$ equals this fiber product.

To do this, we show that $C^{\prime}$ satisfies the universal property of the fiber product. Indeed, let $i_{M}$ and $i_{N}$ be the inclusion morphisms of $C_{\prec}(M)$ and $C_{\prec \text { opp }}(N)$ into $\mathbb{H}_{\mathbf{c}}(H)$. Suppose two morphisms $\phi_{M}: \operatorname{Spec}(R) \rightarrow C_{\prec}(M)$ and $\phi_{N}: \operatorname{Spec}(R) \rightarrow$ $C_{\prec \text { opp }}(N)$ satisfy $i_{M} \circ \phi_{M}=i_{N} \circ \phi_{N}$. Let $\phi: \operatorname{Spec}(R) \rightarrow \operatorname{Spec}\left(K\left[T_{+}(M), T_{-}(N)\right]\right)$ be the product $\phi_{M} \times \phi_{N}$. We will show that $\phi^{*}\left(F_{(n, s)}\right)=0$ for all pairs $(n, s)$, which shows that $\phi$ factors through $C^{\prime}$ with $\phi_{M}=p_{1} \circ \phi$ and $\phi_{N}=p_{2} \circ \phi$, as required.

To see this, let $I_{M} \subseteq R[x, y]$ be the ideal of the pull-back of the universal family on $C_{\prec}(M)$ to $\operatorname{Spec}(R)$, and let $I_{N} \subseteq R[x, y]$ be the ideal of the pull-back of the universal family on $C_{\prec \text { opp }}(N)$ to $\operatorname{Spec}(R)$. The ideal $I_{M}$ is generated by the polynomials $\phi_{M}^{*}\left(g_{i}\right)$ and the ideal $I_{N}$ is similarly generated by $\phi_{N}^{*}\left(f_{i}\right)$. Our assumption that the induced maps to $\mathbb{H}_{\mathbf{c}}(H)$ coincide imply that $I_{M}=I_{N}$.

Fix a minimal generator $n=n_{i}$ of $N$. Let $\mathcal{N}_{i}=\left\{n^{\prime} \in \operatorname{Mon}(K[x, y]) \mid n^{\prime} \prec^{\text {opp }}\right.$ $n_{i}$ and $\left.\operatorname{deg}\left(n^{\prime}\right)=\operatorname{deg}\left(n_{i}\right)\right\}$.

Then for the generator $\tilde{f}_{i}$ of $I_{\prec \text { opp }}(N)$, we have

$$
\begin{aligned}
\tilde{f}_{i} & =n_{i}+\sum_{n \in \mathcal{N}_{i}}\left(\sum_{\substack{\mathcal{P} \text { path from } \\
n_{i} \text { to } n \text { for } \prec^{\mathrm{opp}}}} a_{\mathcal{P}}\right) n \\
& =\sum_{\substack{s \text { standard monomial } \\
\text { for } M, \operatorname{deg}(s)=\operatorname{deg}\left(n_{i}\right)}}\left(\sum_{\substack{\mathcal{H} \text { hike from } \\
n_{i} \text { to } s}} a_{\mathcal{H}}\right) s+g,
\end{aligned}
$$

where $g \in I_{\prec}(M)$.

By the definition of the multigraded Hilbert scheme, the $R$-module $\left(R[x, y] / I_{M}\right)_{a}$ is locally free of rank $H(a)$, where $a=\operatorname{deg}\left(n_{i}\right)$. Since $M \in \mathbb{H}_{\mathbf{c}}(H)$, there are $H(a)$ standard monomials of $M$ of degree $a$. We claim that the standard monomials $s$ of $M$ of degree $a$ span $\left(R[x, y] / I_{M}\right)_{a}$. If not, there is $\tilde{f}=\sum c_{m} m \in R[x, y]_{a}$, where $c_{m} \in R$ and the $m$ are monomials that are not in the span of the standard monomials modulo $I_{m}$. We can choose this polynomial to have $m^{\prime}=\max _{\prec}\left\{m: c_{m} \neq 0, m \in M\right\}$ as small as possible. Choose $i$ so that $m_{i}$ divides $m^{\prime}$. Then $\tilde{f}-c_{m^{\prime}} m^{\prime} / m_{i} \phi_{M}^{*}\left(g_{i}\right)$ is a polynomial with smaller such maximum, so can be written in the desired form. But then the fact that $\phi_{M}^{*}\left(g_{i}\right) \in I_{M}$ gives a contradiction. For a prime $P$ of $R$ the $R_{P}$-module $\left(R_{P}[x, y] / R_{P} I_{M}\right)_{a}$ is free of rank $H(a)$, so the spanning monomials must 
be a basis, and thus have no relations between them. Since $\phi_{N}^{*}\left(\tilde{f}_{i}\right) \in I_{N}=I_{M}$ and $\phi_{M}^{*}(g) \in I_{M}$, we have $\sum \phi^{*}\left(F_{\left(n_{i}, s\right)}\right) s \in I_{M}$, where the sum is as above over the set of $s \notin M$ with $\operatorname{deg}(s)=\operatorname{deg}\left(n_{i}\right)$.

This means that the image of $\sum \phi^{*}\left(F_{n_{i}, s}\right) s \in R_{P}[x, y]$ in each localization at prime $P$ of $R$ must vanish, and so $\sum \phi^{*}\left(F_{n_{i}, s}\right) s=0$ in $R[x, y]$. This means that $\phi^{*}\left(F_{n_{i}, s}\right)=0$ as required.

Example 4.16. Let $M=\left\langle x^{8}, x^{5} y, x^{3} y^{3}, y^{4}\right\rangle$ be the ideal from Example 4.11 and let $N=\left\langle x^{8}, x^{5} y, x^{2} y^{2}, y^{6}\right\rangle$. We order the generators for $N$ with respect to $\prec^{\text {opp }}$, and denote the significant arrows by by $\widetilde{c}_{i}^{\ell}$. We have $n_{0}=y^{6}, n_{1}=x^{2} y^{2}, n_{2}=x^{5} y, n_{3}=x^{8}$, and the significant arrows are $\widetilde{c}_{1}^{1}, \widetilde{c}_{1}^{2}, \widetilde{c}_{2}^{4}$. So the paths from $x^{5} y$ are $\widetilde{c}_{1}^{1}, \widetilde{c}_{1}^{2}, \widetilde{c}_{2}^{4}$. Then

$$
F_{\left(x^{5} y, x^{6}\right)}=c_{1}^{1}+\widetilde{c_{1}^{2}} c_{2}^{3}+\widetilde{c_{1}^{2}} c_{2}^{1} c_{1}^{1} c_{1}^{1}+\widetilde{c_{2}^{4}} S_{x y^{5}, x^{6}}
$$

Not all polynomials look this complicated. For example, $F_{\left(x^{2} y^{2}, x y^{3}\right)}=\widetilde{c}_{1}^{1}+\widetilde{c}_{1}^{2}\left(c_{3}^{1}+\right.$ $\left.c_{2}^{1}+c_{1}^{1}\right)$.

Remark 4.17. There can be significant cancellation in the equations $F_{(n, s)}$ for $E(M, N)$ given in Theorem 4.15. It would be interesting to have a positive formula for these polynomials. For example, this would let us approach the question, asked in [AS05], of whether the T-graph depends on the characteristic of the field when $K$ is algebraically closed.

\section{EXAMPLES}

In this section we consider four different examples of $T$-graphs on multigraded Hilbert schemes.

\subsection{The $T$-graph of $\operatorname{Hilb}^{4}\left(\mathbb{A}^{2}\right)$. .}

The first nontrivial case of the $T$-graph of a multigraded Hilbert scheme is the Hilbert scheme of four points in $\mathbb{A}^{2}$. The torus-fixed points in this case correspond to monomial ideals in $K[x, y]$ with four standard monomials, or equivalently to partitions of four. There are five such ideals/partitions:

(1) $4:\left\langle x^{4}, y\right\rangle$,

(2) $3+1:\left\langle x^{3}, x y, y^{2}\right\rangle$,

(3) $2+2:\left\langle x^{2}, y^{2}\right\rangle$

(4) $2+1+1:\left\langle x^{2}, x y, y^{3}\right\rangle$,

(5) $1+1+1+1:\left\langle x, y^{4}\right\rangle$.

Using this numbering, the edges of this graph are then

$$
\{(1,2),(1,3),(1,5),(2,3),(2,4),(3,4),(3,5),(4,5)\}
$$

Most of these edges are one-dimensional. We list them in the following table, where the parameter $a$ can be any nonzero element of $K$. 


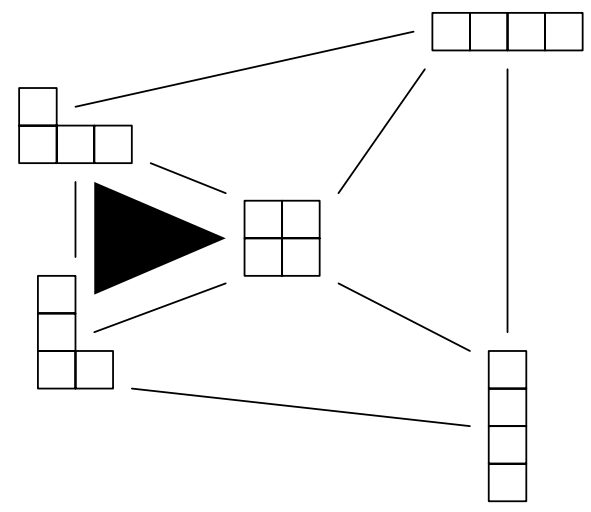

FiguRE 5. The $T$-graph of 4 points in $\mathbb{A}^{2}$.

\begin{tabular}{lll} 
Edge & $\mathbf{c}$ & ideal \\
\hline$(1,2)$ & $(1,-3)$ & $\left\langle x^{4}, y-a x^{3}\right\rangle$ \\
$(1,3)$ & $(1,-2)$ & $\left\langle x^{4}, y-a x^{2}\right\rangle$ \\
$(1,5)$ & $(1,-1)$ & $\left\langle x^{4}, y-a x\right\rangle$ \\
$(2,3)$ & $(1,-1)$ & $\left\langle x^{3}, x y-a x^{2}, y^{2}\right\rangle$ \\
$(3,4)$ & $(1,-1)$ & $\left\langle x^{2}, y^{2}-a x y\right\rangle$ \\
$(3,5)$ & $(2,-1)$ & $\left\langle x^{2}, y^{2}-a x\right\rangle$ \\
$(4,5)$ & $(3,-1)$ & $\left\langle x^{2}, x y, y^{3}-a x\right\rangle$
\end{tabular}

The interesting edge is the one joining the second and fourth ideals indicated by the black triangle. It consists of the ideals

$$
\left\langle x^{3}, x y-a x^{2}, y^{2}-\left(a^{2}+b\right) x^{2}\right\rangle,
$$

for any value of $a, b \in K^{*}$ with $a \neq b^{2}$. Note that the edges $(2,3)$ and $(3,4)$ live in the closure of this edge (by letting $b=-a^{2}$ or $a \rightarrow \infty$ respectively). This is shown in Figure 5 .

5.2. The $T$-graph of two points in $\mathbb{P}^{2}$. We now consider the case of the Hilbert scheme of two points in $\mathbb{P}^{2}$. As a multigraded Hilbert scheme this corresponds to requiring the Hilbert function to be $h(0)=1, h(1)=3$, and $h(i)=2$ for $i \geqslant 2$ for ideals in $K\left[x_{0}, x_{1}, x_{2}\right]$. The $T$-graph has nine vertices, which we label by the saturations of the corresponding monomial ideals, as these have fewer generators. Explicitly, for an ideal $M \in \mathrm{Hilb}_{S}^{h}$, we label the vertex by $\left(M:\left\langle x_{0}, x_{1}, x_{2}\right\rangle^{\infty}\right)$. We can recover $M$ from its saturation by taking the ideal generated by the degree-two part of the saturation. The nine saturated ideals are:

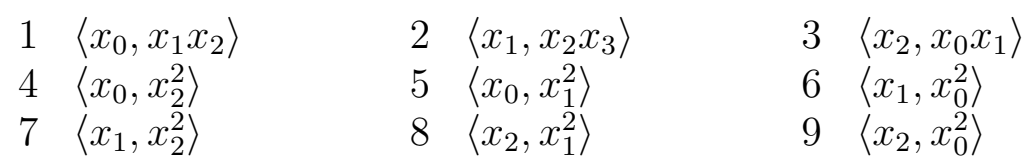

The $T$-graph then has 18 edges. Up to the $S_{3}$-symmetry, these are the pairs: $\left\{\left(\left\langle x_{0}, x_{1} x_{2}\right\rangle,\left\langle x_{1}, x_{0} x_{2}\right\rangle\right),\left(\left\langle x_{0}, x_{1} x_{2}\right\rangle,\left\langle x_{0}, x_{1}^{2}\right\rangle\right),\left(\left\langle x_{0}, x_{1}^{2}\right\rangle,\left\langle x_{1}, x_{0}^{2}\right\rangle\right),\left(\left\langle x_{0}, x_{1}^{2}\right\rangle,\left\langle x_{0}, x_{2}^{2}\right\rangle\right)\right.$, $\left.\left(\left\langle x_{0}, x_{1}^{2}\right\rangle,\left\langle x_{2}, x_{1}^{2}\right\rangle\right)\right\}$. This is shown in Figure6. The shaded triangles indicate that the edges joining ideals 4 and 5, joining 6 and 7, and joining 8 and 9 are two dimensional, and have the third vertex of the respective triangles in their closure. For example, 


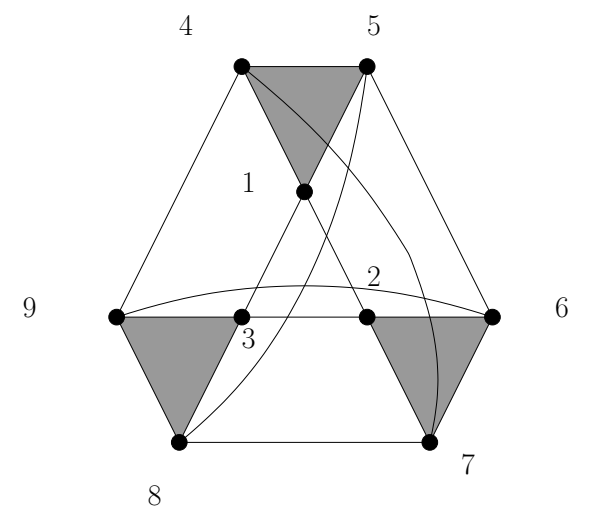

FiguRE 6 . The $T$-graph of 2 points in $\mathbb{P}^{2}$.

ideals in the edge joining vertices 4 and 5 have the form $\left\langle x_{0}^{2}, x_{0} x_{1}, x_{0} x_{2}, x_{1}^{2}+a x_{1} x_{2}+\right.$ $\left.b x_{2}^{2}\right\rangle$ for $a, b \in K$ with $b \neq 0$.

\subsection{The $T$-graph of $\operatorname{Hilb}^{8}\left(\mathbb{A}^{4}\right)$. .}

The Hilbert scheme of 8 point in $\mathbb{A}^{4}$ has two irreducible components, of dimensions 32 and 25, which intersect in a scheme of dimension 24. See [CEVV09] for more details. The $T$-graph of this Hilbert scheme has 684 vertices, and 9278 edges. All vertices lie on the component of dimension 32. This data can be found in the package TEdges Mac11].

5.4. Small Hilbert schemes of points in the plane. We list here the data for the Hilbert schemes $\operatorname{Hilb}^{d}\left(\mathbb{A}^{2}\right)$ for small values of $d$. This illustrates the use of our necessary condition in this range.

\begin{tabular}{lllllll}
$d$ & \# ideals & \# pairs & \# pairs & \# pairs $(M, N)$ & \# pairs $(M, N)$ & \# edges \\
& $M$ & $(M, N)$ & $M<N$ & $\begin{array}{l}\text { with an arrow } \\
\text { map }\end{array}$ & $\begin{array}{l}\text { with an arrow map } \\
\text { on the duals }\end{array}$ & \\
\hline 4 & 5 & 10 & 8 & 8 & 8 & 8 \\
5 & 7 & 21 & 15 & 15 & 15 & 15 \\
6 & 11 & 55 & 37 & 37 & 37 & 37 \\
7 & 15 & 105 & 55 & 52 & 52 & 52 \\
8 & 22 & 231 & 100 & 99 & 99 & 99 \\
9 & 30 & 435 & 170 & 166 & 166 & 286 \\
10 & 42 & 861 & 291 & 280 & 280 & 401 \\
11 & 56 & 1540 & 411 & 401 & 401 & 663 \\
12 & 77 & 2926 & 688 & 663 & 663 & 140 \\
13 & 101 & 5050 & 957 & 918 & 918 & 2076 \\
14 & 135 & 9045 & 1524 & 1446 & 1446 & 3031 \\
15 & 176 & 15400 & 2203 & 2076 & 2076 & 3031 \\
16 & 231 & 26565 & 3218 & 3033 & &
\end{tabular}

Remark 5.1. This table was created with the Macaulay 2 package TEdges [Mac11]. For $d \leqslant 15$ the edge code was run independently from the partial order and arrowmap code, so the containment of the set of edges in the set for which there exist arrow-maps gives a check on the code. This was not possible for $d>15$ for memory 
usage reasons. While this table shows that the necessary conditions of Theorem 1.3 are sufficient for small $d$ in $\mathrm{Hilb}^{d}\left(\mathbb{A}^{2}\right)$, we caution that 16 points is still comparatively small for this problem, so do not regard this as strong evidence of the condition being sufficient, particularly in light of Example 3.6.

\section{REFERENCES}

[AS05] Klaus Altmann and Bernd Sturmfels. The graph of monomial ideals. J. Pure Appl. Algebra, 201(1-3):250-263, 2005.

[BBCM02] Andrzej Białynicki-Birula, James B. Carrell, and W. Monty McGovern. Algebraic quotients. Torus actions and cohomology. The adjoint representation and the adjoint action, volume 131 of Encyclopaedia of Mathematical Sciences. Springer-Verlag, Berlin, 2002. Invariant Theory and Algebraic Transformation Groups, II.

[BCS08] Tom Braden, Linda Chen, and Frank Sottile. The equivariant Chow rings of Quot schemes. Pacific J. Math., 238(2):201-232, 2008.

[BH93] Winfried Bruns and Jürgen Herzog. Cohen-Macaulay rings, volume 39 of Cambridge Studies in Advanced Mathematics. Cambridge University Press, Cambridge, 1993.

[CEVV09] Dustin A. Cartwright, Daniel Erman, Mauricio Velasco, and Bianca Viray. Hilbert schemes of 8 points. Algebra Number Theory, 3(7):763-795, 2009.

[CLO07] David Cox, John Little, and Donal O'Shea. Ideals, varieties, and algorithms. Undergraduate Texts in Mathematics. Springer, New York, third edition, 2007. An introduction to computational algebraic geometry and commutative algebra.

[Eis95] David Eisenbud. Commutative algebra, volume 150 of Graduate Texts in Mathematics. Springer-Verlag, New York, 1995. With a view toward algebraic geometry.

[Eva02] Laurent Evain. Incidence relations among the Schubert cells of equivariant punctual Hilbert schemes. Math. Z., 242(4):743-759, 2002.

[Eva04] Laurent Evain. Irreducible components of the equivariant punctual Hilbert schemes. Adv. Math., 185(2):328-346, 2004.

[Eva07] Laurent Evain. The Chow ring of punctual Hilbert schemes on toric surfaces. Transform. Groups, 12(2):227-249, 2007.

[GKM98] Mark Goresky, Robert Kottwitz, and Robert MacPherson. Equivariant cohomology, Koszul duality, and the localization theorem. Invent. Math., 131(1):25-83, 1998.

[GS] Daniel R. Grayson and Michael E. Stillman. Macaulay2, a software system for research in algebraic geometry. Available at http://www.math.uiuc.edu/Macaulay2/.

[Hai98] Mark Haiman. t, q-Catalan numbers and the Hilbert scheme. Discrete Math., 193(13):201-224, 1998. Selected papers in honor of Adriano Garsia (Taormina, 1994).

[Har66] Robin Hartshorne. Connectedness of the Hilbert scheme. Inst. Hautes Études Sci. Publ. Math., (29):5-48, 1966.

[HS04] Mark Haiman and Bernd Sturmfels. Multigraded Hilbert schemes. J. Algebraic Geom., 13(4):725-769, 2004.

[Ive72] Birger Iversen. A fixed point formula for action of tori on algebraic varieties. Invent. Math., 16:229-236, 1972.

[Mac11] Diane Maclagan. TEdges, a Macaulay2 package to compute the $T$-graph of a multigraded Hilbert scheme. Available at http://www.warwick.ac.uk/staff/D.Maclagan/papers/TEdges.html, 2011.

[MS05] Ezra Miller and Bernd Sturmfels. Combinatorial commutative algebra, volume 227 of Graduate Texts in Mathematics. Springer-Verlag, New York, 2005.

[MS10] Diane Maclagan and Gregory G. Smith. Smooth and irreducible multigraded Hilbert schemes. Adv. Math., 223(5):1608-1631, 2010.

[Mum62] David Mumford. Further pathologies in algebraic geometry. Amer. J. Math., 84:642-648, 1962.

[Nak99] Hiraku Nakajima. Lectures on Hilbert schemes of points on surfaces, volume 18 of University Lecture Series. American Mathematical Society, Providence, RI, 1999. 
[Nak01] Iku Nakamura. Hilbert schemes of abelian group orbits. J. Algebraic Geom., 10(4):757$779,2001$.

[PS02] Irena Peeva and Mike Stillman. Toric Hilbert schemes. Duke Math. J., 111(3):419-449, 2002.

[PS05] Irena Peeva and Mike Stillman. Connectedness of Hilbert schemes. J. Algebraic Geom., 14(2):193-211, 2005.

[San05] Francisco Santos. Non-connected toric Hilbert schemes. Math. Ann., 332(3):645-665, 2005.

[Yam94a] Joachim Yaméogo. Décomposition cellulaire de variétés paramétrant des idéaux homogènes de $\mathbf{C} \llbracket x, y \rrbracket$. Incidence des cellules. I. Compositio Math., 90(1):81-98, 1994.

[Yam94b] Joachim Yaméogo. Décomposition cellulaire de variétés paramétrant des idéaux homogènes de $\mathbf{C} \llbracket x, y \rrbracket$. Incidence des cellules. II. J. Reine Angew. Math., 450:123-137, 1994.

Department of Mathematics, University of Connecticut, 196 Auditorium Road, StOrRs CT 06269, USA

E-mail address: milena.hering@uconn.edu

Mathematics Institute, Zeeman Building, University of Warwick, Coventry CV4 7AL, United Kingdom

E-mail address: D.Maclagan@warwick.ac.uk 